\title{
EGF and curcumin co-encapsulated nanoparticle/ hydrogel system as potent skin regeneration agent
}

This article was published in the following Dove Press journal:

International Journal of Nanomedicine

17 August 2016

Number of times this article has been viewed

\author{
Xiaoling $\mathrm{Li}^{1}$ \\ Xianlong $\mathrm{Ye}^{2}$ \\ Jianying $\mathbf{Q i}^{2}$ \\ Rangrang Fan' \\ Xiang Gao' \\ Yunzhou $\mathrm{Wu}^{2}$ \\ Liangxue Zhou' \\ Aiping Tong' \\ Gang Guo'
}

'State Key Laboratory of Biotherapy and Cancer Center, Department of Neurosurgery, West China Hospital, Sichuan University and Collaborative Innovation Center for Biotherapy, Chengdu, People's Republic of China; ${ }^{2}$ College of Life Science, Northeast Agriculture University, Harbin, People's Republic of China
Correspondence: Gang Guo State Key Laboratory of Biotherapy and Cancer Center, Department of Neurosurgery, West China Hospital, Sichuan University and Collaborative Innovation Center for Biotherapy, No 17, Block 3, Southern Renmin Road, Chengdu, Sichuan 6I004I, People's Republic of China

Tel +862885I64063

$\mathrm{Fax}+862885164060$

Email guogang@scu.edu.cn

Yunzhou Wu

College of Life Science, Northeast Agriculture University, No 59 Wood Street, Xiangfang District, Harbin, Heilongjiang 150030, People's Republic of China

Email wuyunzhou@neau.edu.cn

\begin{abstract}
Wound healing is a complex multifactorial process that relies on coordinated signaling molecules to succeed. Epidermal growth factor (EGF) is a mitogenic polypeptide that stimulates wound repair; however, precise control over its application is necessary to reduce the side effects and achieve desired therapeutic benefits. Moreover, the extensive oxidative stress during the wound healing process generally inhibits repair of the injured tissues. Topical applications of antioxidants like curcumin (Cur) could protect tissues from oxidative damage and significantly improve tissue remodeling. To achieve much accelerated wound healing effects, we designed a novel dual drug co-loaded in situ gel-forming nanoparticle/hydrogel system (EGF-Cur-NP/H) which acted not only as a supportive matrix for the regenerative tissue, but also as a sustained drug depot for EGF and Cur. In the established excisional full-thickness wound model, EGFCur-NP/H treatment significantly enhanced wound closure through increasing granulation tissue formation, collagen deposition, and angiogenesis, relative to normal saline, nanoparticle/ hydrogel (NP/H), Cur-NP/H, and EGF-NP/H treated groups. In conclusion, this study provides a biocompatible in situ gel-forming system for efficient topical application of EGF and Cur in the landscape of tissue repair.
\end{abstract}

Keywords: epidermal growth factor, EGF, curcumin, wound healing, thermosensitive hydrogel, nanoparticles

\section{Introduction}

Dermal injury, including trauma, surgical incisions, and burns, continue to be one of the most prevalent and socioeconomically burdensome health care issues in the world. ${ }^{1}$ Since the skin serves as a protective barrier against the outside world, when injured, the subcutaneous tissues and organs are threatened by pathogens and excessive water loss. Wound repair is a complex and dynamic process requiring the collaborative efforts of soluble mediators, blood cells, extracellular matrix, and parenchymal cells. ${ }^{2-4}$ Numerous growth factors, such as epidermal growth factor (EGF), transforming growth factor beta (TGF- $\beta$ ), fibroblast growth factor (FGF), and platelet-derived growth factor (PDGF), were considered the key regulators of the wound healing process. ${ }^{5-7}$ It has been established that impaired chronic wound healing is associated with decreased secretion of endogenous growth factors. Therefore, provision of exogenous growth factors at precisely timed intervals would provide stimulation to induce faster reepithelialization, as well as reduce the risk of infection. ${ }^{8-10}$

Unfortunately, the therapeutic use of growth factors is limited since they can be easily degraded by proteinases or removed by exudate before reaching the wound bed. Few growth factors, except PDGF-BB, have successfully completed clinical trials. ${ }^{11}$ To overcome the low stability in vivo and undesirable effects at high systemic levels, growth factors need to be applied in a sustained and localized fashion, so as to achieve 
maximum therapeutic benefits with minimal patient risks. ${ }^{12}$ Furthermore, combination therapy of multiple agents with different mechanisms can act synergistically and maximize therapeutic effects, and is considered to be a potential strategy for the effective treatment of many diseases. ${ }^{13}$ Hence, we hypothesized that the combination of growth factors with other chemotherapy drugs may greatly accelerate wound closure.

EGF, a small polypeptide with molecular mass of $6 \mathrm{kDa}$, has been first and most successfully applied for wound repair. It accelerates wound healing rates through enhancing synthesis of basement membrane and extracellular matrix components, cell motility, and proliferation. ${ }^{14,15}$ Yet, the halflife of EGF in the body is too short to exert the biological activity effectively when applied via injection or in free form. In addition, accumulating evidences suggested that EGF is effective as an adjuvant but not as monotherapy for wound repair mainly due to its ineffective delivery and rapid proteolysis. ${ }^{16}$ Curcumin (Cur) is a natural yellow pigment and the major active ingredient of turmeric which has been widely used for centuries in Indian and Chinese medicine for the treatment of various inflammatory conditions and chronic diseases. ${ }^{17}$ Accumulating data show that Cur is a promising wound healing agent when used topically because of its strong anti-oxidant, anti-inflammatory, and anti-infective activities. ${ }^{18,19}$ However, the in vivo bioavailability of Cur is very low, mainly associated with its poor aqueous solubility and rapid degradation in physiological buffer by hydrolysis. ${ }^{20}$ So it requires the development of suitable carrier vehicles to enhance stability and solubility of Cur and deliver the molecule in a sustained manner at therapeutic levels.

With this in mind, we proposed a novel dual drug loaded in situ gel-forming nanoparticle/hydrogel system (EGF-Cur$\mathrm{NP} / \mathrm{H}$ ) for cutaneous wound healing. The copolymers used for formulation of the vehicle were synthesized with the monomers $_{L}$-lactic acid ( - LA) and reverse Pluronic ${ }^{\circledR} 10 R 5$ (10R5) via ring-opening copolymerization method. Normal and reverse pluronic triblock copolymers with polyethylene glycol (PEG)-polypropylene glycol (PPG)-PEG and PPG-PEG-PPG block sequences respectively are widely applied in biomedical and pharmaceutical fields for their unique solution behavior, self-assembling ability, and lowtoxicity. ${ }^{21,22}$ Pluronics like 10R5 and F127 are approved by the US Food and Drug Administration as drug excipient. However, the copolymers have a high critical micelle concentration (CMC), and they suffer from low capacity to encapsulate large amounts of hydrophobic drugs. ${ }^{23}$ As a result, new chemical functional groups have been grafted onto the copolymer blocks to overcome the limitations of pluronics as drug carriers. ${ }^{24}$ Hassanzadeh et al conjugated the hydrophobic quercetin to PPG segments of 10R5, which decreased the CMC value with more than one order of magnitude and the drug encapsulation capacity was considerably enhanced. ${ }^{23}$ Compared to the large number of publications for normal pluronic copolymers, much less attention has been given to reverse pluronics. Thus, it will be interesting to study the chemical structure modification and pharmaceutical applications of reverse pluronic copolymers.

In the present study, EGF and Cur were co-encapsulated in polylactic acid (PLA)-10R5-PLA (number-average molecular weight $\left.\left[M_{n}\right]=19.8 \times 10^{3}\right)$ NPs using the water-inoil-in-water (w/o/w) emulsion solvent evaporation method for the first time. To optimize the topical administration, the EGF and Cur co-loaded NPs (EGF-Cur-NP) were dispersed into the thermosensitive and biocompatible PLA-10R5-PLA $\left(M_{\mathrm{n}}=4.6 \times 10^{3}\right)$ hydrogel to form an in situ gel-forming composite (EGF-Cur-NP/H). And the fast and comprehensive wound healing effects of EGF-Cur-NP/H composite were studied in detail using a full-thickness incision rat model.

\section{Materials and methods}

\section{Materials, cell lines, and animals}

The 10R5 (PPG-PEG-PPG, $\left.M_{\mathrm{n}}=2,000\right)$, Cur (80\% Cur and $20 \%$ other curcuminoids), polyvinyl alcohol (weight-average molecular weight $\left[M_{\mathrm{w}}\right] 30,000-70,000$, degree of hydrolysis $87 \%-90 \%)$, MTT, and stannous octoate $\left[\mathrm{Sn}(\mathrm{Oct})_{2}\right]$ were purchased from Sigma-Aldrich Co. (St Louis, MO, USA). L-LA was provided by Guangshui National Chemical Co. (Guangshui, People's Republic of China). All other chemicals used in this paper were analytical grade.

Human embryonic kidney cell line HEK293 and 3T3 cells were provided by the American Type Culture Collection (ATCC, Manassas, VA, USA). The cells were cultured in Dulbecco's Modified Eagle's Medium (Gibco, Thermo Fisher Scientific, Waltham, MA, USA) with supplements of $10 \%$ fetal bovine serum (Gibco, Thermo Fisher Scientific) and $1 \%$ penicillin/streptomycin in a humidified atmosphere of 5\% $\mathrm{CO}_{2}$. Male Sprague Dawley rats weighing 200-220 g were purchased from HFK Bio-Technology Company (Beijing, People's Republic of China). All animals were treated and used following the Guide for the Care and Use of Laboratory Animals, Institute of Laboratory Animal Resources. All animal experiments were conducted under the approved guidelines of Sichuan University and approved by the Animal Care Committee of Sichuan University (Chengdu, People's Republic of China). 


\section{Synthesis and characterization of PLA-IOR5-PLA block copolymers}

The biodegradable PLA-10R5-PLA block copolymers were synthesized by ring-opening copolymerization method. In brief, calculated amount of ${ }_{\mathrm{L}}$-LA (30 $\mathrm{g}$ for sample $\mathrm{S} 1$ and $45 \mathrm{~g}$ for S2) and 10R5 (20 $\mathrm{g}$ for sample S1 and $5 \mathrm{~g}$ for S2) were added into a dry glass ampoule under nitrogen atmosphere, and catalyzed by $0.15 \mathrm{~g}$ of $\mathrm{Sn}(\mathrm{Oct})_{2}$. The reaction system was kept at $130^{\circ} \mathrm{C}$ for 10 hours and rapidly heated to $140^{\circ} \mathrm{C}$ under vacuum for another 1 hour. After being cooled to room temperature under nitrogen atmosphere, the PLA-10R5-PLA copolymer was firstly dissolved in methylene chloride and then precipitated using excess cold petroleum ether. The mixture was filtered and dried at $40^{\circ} \mathrm{C}$ under vacuum for 48 hours. The purified copolymers were kept in a desiccator before use. ${ }^{1} \mathrm{H}$ nuclear magnetic resonance spectroscopy $\left({ }^{1} \mathrm{H}\right.$ NMR; Bruker Avance III 400, Bruker Optik GmbH, Ettlingen, Germany), Fourier transform infrared spectroscopy (FTIR; Nicolet 200 SXV, Thermo Fisher Scientific) and gel permeation chromatography (GPC; Agilent 110 HPLC, Agilent Technologies, Santa Clara, CA, USA) were used to characterize the obtained copolymers.

\section{Fabrication and characterization of EGF-Cur-NP}

EGF was expressed and purified as per the following method. Briefly, the recombinant $\mathrm{pSUMO}$ vector containing the $\mathrm{hEGF}$ was transformed into host bacterium, BL21 (DE3). Single colonies of Escherichia coli BL21 (containing a recombinant plasmid of pSUMO-hEGF) were grown in Luria-Bertan media containing ampicillin $(100 \mu \mathrm{g} / \mathrm{mL})$. When the optical density ${ }_{600}$ reached approximately 0.35 , isopropyl $\beta$-Dthiogalactopyranoside was added into the medium at the final concentration of $0.5 \mathrm{mmol} / \mathrm{L}$. The culture was then grown at $25^{\circ} \mathrm{C}$ for 6 hours to induce expression of the fusion protein. After that, cells were harvested by centrifugation $(4,000 \mathrm{rpm}$, $4^{\circ} \mathrm{C}, 30$ minutes $)$ and resuspended in binding buffer $(50 \mathrm{mM}$ $\mathrm{Na}_{3} \mathrm{PO}_{4}, 500 \mathrm{mM} \mathrm{NaCl}, 20 \mathrm{mM}$ imidazole, pH 8.0). EGF protein was purified by two-step Ni Sepharose 6 Fast Flow chromatography in an AKTA Purifier system. The purity of the protein was determined by high performance liquid chromatography (HPLC) analysis.

The EGF-Cur-NP was prepared by w/o/w double emulsion solvent evaporation method. ${ }^{25}$ In brief, organic phase, consisting of $1 \mathrm{~mL}$ of dichloromethane/ethyl acetate $(1 / 1, \mathrm{v} / \mathrm{v}), 9 \mathrm{mg}$ of PLA-10R5-PLA (S2, $\left.M_{\mathrm{n}}=19.8 \times 10^{3}\right)$ and $1 \mathrm{mg}$ of Cur, was emulsified with $100 \mu \mathrm{L}$ of EGF aqueous solution $(5 \mathrm{mg} / \mathrm{mL})$ using a probe sonication for
60 seconds at $39.6 \mathrm{~W}$ (TM300 sonic disruptor; Teledyne Tekmar, Mason, OH, USA). Then the o/w emulsion was added dropwise to the external water phase, $4 \mathrm{~mL}$ of $1 \%$ (w/v) polyvinyl alcohol, and sonicated for another $60 \mathrm{sec}-$ onds. The final w/o/w solution was de-solvated by rotary evaporation under vacuum at $37^{\circ} \mathrm{C}$ (Rotavapor R-114; Büchi, Flawil, Switzerland). EGF-NP, Cur-NP, and black NP were prepared using the method as the minor modulation. The NPs were finally washed and collected by centrifugation $(13,300 \mathrm{rpm})$ at $4^{\circ} \mathrm{C}$ for 90 minutes (Eppendorf, Hamburg, Germany), and the supernatant was used for determination of the un-encapsulated EGF and Cur.

A Malvern Nano-ZS 90 (Malvern Instruments, Malvern, UK) laser particle size analyzer was used to determine particle-size distribution and polydispersity index (PDI) of the prepared NPs. The surface morphology of EGF-Cur-NP was examined via a transmission electron microscopy (TEM; H-6009IV; Hitachi Ltd., Tokyo, Japan). The freshly-prepared sample was diluted with distilled water and placed on a copper grid covered with nitrocellulose, then negatively stained with phosphotungstic acid, and dried at room temperature before observation. The samples were also characterized by FTIR.

The amount of un-encapsulated Cur was determined by HPLC (Waters Alliance 2695, Waters, Milford, MA, USA) with a reversed phase $\mathrm{C} 18$ column $(4.6 \times 150 \mathrm{~mm}-5 \mu \mathrm{m}$, Inertsil/WondaSil, Kyoto, Japan). An amount of $20 \mu \mathrm{L}$ of the sample solution was injected and analyzed in the mobile phase consisting of methanol $/ 0.3 \%$ acetic acid $(80 / 20, \mathrm{v} / \mathrm{v})$ at the flow rate of $1.0 \mathrm{~mL} / \mathrm{min}$. The detection wavelength was $420 \mathrm{~nm}$. The amount of EGF was analyzed using enzyme-linked immunosorbent assay (ELISA) following the manufacturer's instructions (Invitrogen, Thermo Fisher Scientific). Drug loading (DL) and encapsulation efficiency (EE) were calculated according to Equation 1 and Equation 2:

$$
\begin{aligned}
& \mathrm{DL}=\frac{\text { Drug }}{\text { Drug }+ \text { polymer }} \times 100 \% \\
& \mathrm{EE}=\frac{\text { Experimental DL }}{\text { Theoretical DL }} \times 100 \%
\end{aligned}
$$

\section{Preparation and characterization of EGF-Cur-NP/H}

Purified PLA-10R5-PLA $\left(M_{\mathrm{n}}=4.6 \times 10^{3}\right)$ copolymer was dissolved in normal saline (NS); then calculated amount of EGF-Cur-NP was added into the copolymer aqueous solution (30 wt $\%$ ) at $25^{\circ} \mathrm{C}$ to form an injectable homogeneous solution 
(EGF-Cur-NP/H). The surface structure of EGF-Cur-NP/H was observed under the scanning electron microscopy (JSM5900LV; JEOL, Tokyo, Japan). Samples were frozen in liquid nitrogen, lyophilized for 24 hours, and sputter-coated with silver before observation. Rheological measurements were carried out by rheometry (AR Rheometer 2000ex; TA Instruments, New Castle, DE, USA) to study the sol-gel transition property as previously reported. ${ }^{24}$

\section{In vitro cytotoxicity assay}

The in vitro cytotoxicity of EGF-Cur-NP/H was determined through standard MTT assays using HEK293 and 3T3 cells. EGF-Cur-NP/H was extracted with the culture medium for 48 hours at $37^{\circ} \mathrm{C}$. The cells were seeded at a density of $5 \times 10^{3}$ per well in a 96-well plate and incubated overnight. The cells were exposed to the leachates of different concentrations $(100 \%, 50 \%, 25 \%, 0 \%)$ and $0.1 \%$ phenol (positive control). At specific time points, $20 \mu \mathrm{L}$ of MTT was added to each well. Four hours later, the precipitated formazan was dissolved in $150 \mu \mathrm{L}$ of dimethyl sulfoxide, and the optical density at $570 \mathrm{~nm}$ was measured. All results were the mean of six test runs.

\section{In vitro drug release profile}

The in vitro release behaviors of EGF and Cur from EGFCur-NP and EGF-Cur-NP/H were determined by a modified dialysis method. An amount of $500 \mu \mathrm{L}$ of free EGF, free Cur, EGF-Cur-NP and EGF-Cur-NP/H aqueous solution were placed into the dialysis bags with a molecular weight cutoff of $14 \mathrm{kDa}$ and immersed in $10 \mathrm{~mL}$ phosphate-buffered saline (PBS) ( $\mathrm{pH} 7.4)$ containing Tween $80(0.5 \% \mathrm{v} / \mathrm{v})$. Samples were then gently shaken at $37^{\circ} \mathrm{C}$ in a water bath at $100 \mathrm{rpm}$. At specific time intervals, all media were removed and replaced by fresh media. The concentration of EGF and Cur were quantified via ELISA and HPLC, respectively, as mentioned earlier. Three independent experiments were performed.

\section{In vivo wound healing assay}

Full-thickness excision wounds of $2 \times 2 \mathrm{~cm}^{2}$ were created under ketamine hydrochloride (60 $\mathrm{mg} / \mathrm{kg}$ body weight) anesthesia. Then, the rats were randomly assigned to one of the following groups (12 rats per group): NS, NP-H, EGF-NP/H (containing $50 \mu \mathrm{g}$ EGF), Cur-NP/H (containing $100 \mu \mathrm{g}$ Cur), and EGF-Cur-NP/H (containing $25 \mu \mathrm{g}$ EGF and $50 \mu \mathrm{g}$ Cur). The wounds were topically treated with a single application of the aforementioned composites and covered with a piece of Tegaderm ${ }^{\circledR}(3 \mathrm{M}$, Maplewood, MN, USA) to prevent the rats from removing the treatments. Subsequent measurement of wound area was taken on days $3,7,10$, and 14 post-wounding, and wound closure was calculated according to Equation 3:

$$
\text { Wound closure } \%=\frac{A_{0}-A_{t}}{A_{0}} \times 100 \%
$$

where $A_{0}$ was the original wound area on day 0 , and $A_{t}$ was the wound area on day $t$ post-wounding.

On days 7 and 14, six animals from each group were sacrificed to collect the wounds along with the surrounding tissue which were bisected into two halves for further study. One portion of the tissue halves collected on day 7 and 14 were fixed by paraformaldehyde (4\% in PBS) for 48 hours and then embedded in paraffin, and sectioned. The other wound halves harvested on day 14 were embedded in TissueTek O.C.T. compound (Sakura Finetek, Torrance, CA, USA) and stored at $-80^{\circ} \mathrm{C}$ until further processing.

\section{Histopathological and immunofluorescence analysis}

Paraffin sections of $4 \mu \mathrm{m}$ were stained with hematoxylin and eosin (H\&E) to assess the gross histopathological changes at the wound site. Histological scorings of granulation tissue formation and wound maturity were performed using a 5-point visual scoring scale by two independent investigators in a blinded fashion as per the previously reported method. ${ }^{26}$ The extent of collagen deposition in the wound tissue was studied by Masson's trichrome (MT) stain. And semi-quantitative estimation of collagen was carried out by measuring the blue area percentage of the wound tissue by using Image Pro Plus 6.0 software (Media Cybernetics, Rockville, MD, USA).

Immunostaining was performed on paraffin sections of the wounds collected at day 7 post-wounding to determine the expression of TGF- $\beta 1$. Rabbit polyclonal anti-TGF- $\beta 1$ antibody (1:100; Santa Cruz Biotechnology Inc., Dallas, TX, USA) was used as the primary antibody. The green area percentage of the stained tissue was measured for semi-quantitative assay of TGF- $\beta 1$ expression. Furthermore, the frozen sections of $6 \mu \mathrm{m}$ were stained with rat polyclonal antibody against mouse CD31 (1:100, BD Pharmingen ${ }^{\mathrm{TM}}$; BD Biosciences, San Jose, CA, USA) following the manufacturer's instruction to determine the vascular density. The stained samples were visualized under an optical microscope (Olympus, BX 45; Olympus Corporation, Tokyo, Japan). All histological analyses were performed on six wounds per group. 


\section{Statistical analysis}

Data were analyzed using the software program Origin Pro 7.5. Comparisons of experimental data in different groups were performed using one-way analysis of variance (Stat View). All data were expressed as the mean \pm standard deviation (SD), and a $P$-value $<0.05$ was considered statistically significant.

\section{Results}

\section{Characterization of the PLA-I0R5-PLA copolymer}

The ring-opening polymerization method as indicated in Figure 1A was used to synthesize the PLA-10R5PLA copolymers. Figure 1B shows the FTIR spectra of 10R5 and PLA-10R5-PLA. The molecular weight and distribution of the copolymers were detected by GPC (Figure 1C). The molecular weight of the PLA-10R5-PLA copolymers was also determined by ${ }^{1} \mathrm{H}$ NMR. The curves are presented in Figure $\mathrm{S} 1$ and the data are summarized in Table 1.

\section{Characterization of EGF-Cur-NP}

The purity of EGF was determined by HPLC analysis as shown in Figure S2. In this study, EGF-Cur-NP with quite high DL and EE was prepared using the w/o/w emulsion solvent evaporation method. As shown in Figure 2A, Cur formed a turbid suspension in water due to its poor water solubility. In contrast, clear solutions of Cur-NP and EGF-Cur-NP could be observed. The size, DL, and EE of the prepared NPs are shown in Table 2. The EGF-Cur-NP had a larger particle size of $145.3 \pm 2.8 \mathrm{~nm}$ compared with the blank NP (123.4 $\pm 3.8 \mathrm{~nm})$, Cur-NP $(135.3 \pm 3.2 \mathrm{~nm})$, and EGF-NP $(131.3 \pm 4.5 \mathrm{~nm})$ (Figures 2B and S3). In addition, the TEM image (Figure 2C) suggested that EGF-Cur-NPs were spherical and monodispersed. Figure 2D showed that the characteristic peaks of EGF, Cur, and the black NP were also recorded in the spectrum of EGF-Cur-NP, which suggested that EGF and Cur were successfully co-encapsulated in the PLA-10R5-PLA copolymer NPs.

\section{Characterization of EGF-Cur-NP/H}

Both the black hydrogel and EGF-Cur-NP/H exhibited temperature-dependent sol-gel phase transition. The aqueous

A

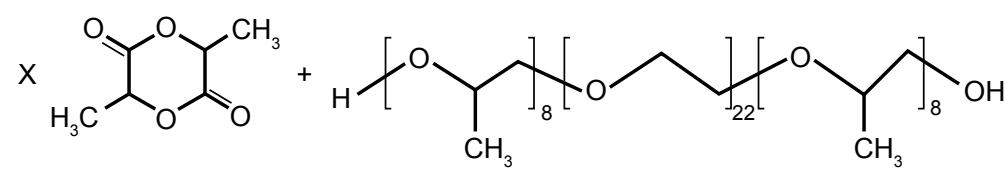<smiles>[X]C(C)(O)C(C)C(=O)C(C)(C)OC(C)CC(C)OCCC(C)(C)OC(C)CC(C)(C)OC(C)(C)C(=O)C(C)O</smiles>

B

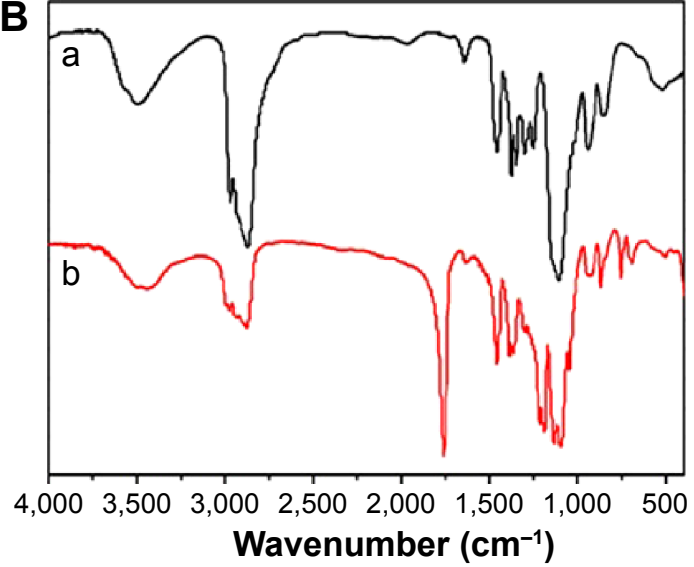

C

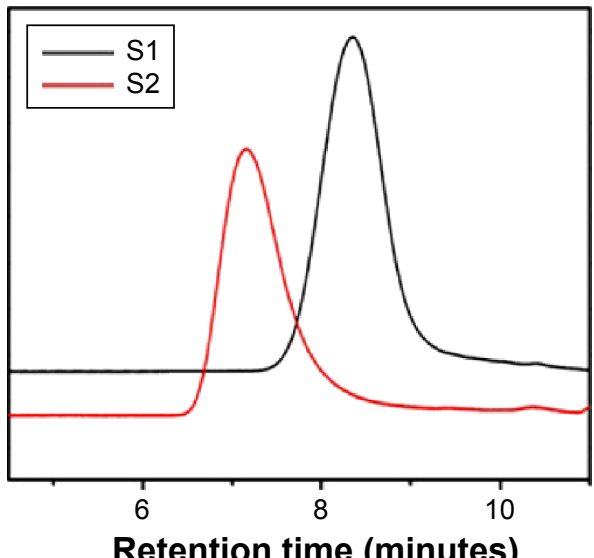

Figure I Synthesis and characterization of PLA-IOR5-PLA copolymer.

Notes: (A) Synthesis scheme of the PLA-I0R5-PLA block copolymer; (B) FTIR spectrum (KBr) of reverse pluronic ${ }^{\circledR}$ IOR5 (a) and copolymer SI, PLA-I0R5-PLA, $\mathrm{M}_{\mathrm{n}}=4.6 \times 10^{3}$ (b); (C) GPC curves of the prepared PLA-I0R5-PLA block copolymers, $\mathrm{SI}, \mathrm{M}_{\mathrm{n}}=4.6 \times 10^{3}, \mathrm{~S} 2, \mathrm{M}_{\mathrm{n}}=19.8 \times 10^{3}$.

Abbreviations: PLA, polylactic acid; IOR5, reverse Pluronic ${ }^{\circledR}$ IOR5; FTIR, Fourier transform infrared spectroscopy; GPC, gel permeation chromatography; $M_{n}$, numberaverage molecular weight; $\mathrm{Sn}(\mathrm{Oct})_{2}$, stannous octoate. 
Table I The PLA-IOR5-PLA copolymers synthesized in this work

\begin{tabular}{lllllll}
\hline Code & PLA:I OR5 $(w / w)^{a}$ & $M_{n}{ }^{a}\left(\times 10^{3}\right)$ & $M_{n}{ }^{b}\left(\times 10^{3}\right)$ & $M_{n}{ }^{c}\left(\times 10^{3}\right)$ & $M_{w}{ }^{c}\left(\times 10^{3}\right)$ & $M_{w} / M_{n}{ }^{c}$ \\
S1 & $3,000: 2,000$ & 5 & 4.6 & 4.1 & 1.5 & 1.35 \\
S2 & $18,000: 2,000$ & 20 & 19.8 & 15.9 & 26.9 & 1.69 \\
\hline
\end{tabular}

Notes: aTheoretical value, calculated from feed ratio; bcalculated according to 'H NMR results; 'cdetermined by GPC in THF (polystyrene standard). SI, $M_{n}=4.6 \times 10^{3}, \mathrm{~S} 2$, $M=19.8 \times 10^{3}$.

Abbreviations: PLA, polylactic acid; IOR5, reverse Pluronic ${ }^{\circledast}$ IOR5; $M_{\mathrm{n}}$, number-average molecular weight; $M_{w^{\prime}}$, weight-average molecular weight; GPC, gel permeation chromatography; 'H NMR, 'H nuclear magnetic resonance spectroscopy; THF, tetrahydrofuran.

solutions were free-flowing sol at $25^{\circ} \mathrm{C}$, but formed solid-like gel when heated to $37^{\circ} \mathrm{C}$ (Figure $3 \mathrm{~A}$ ). As shown in Figure 3B, the EGF-Cur-NP/H possessed a porous 3D structure where the irregular pores are interconnected. Rheological property of EGF-Cur-NP/H in Figure 3C confirmed that the composite exhibited sol-gel transition at the physiological temperature, approximately $37^{\circ} \mathrm{C}$.

\section{In vitro cytotoxicity assay}

MTT assays were also used to determine the cytotoxicity of EGF-Cur-NP/H (Figure 4A and B). The cell viability of the HEK293 and 3T3 cells slightly decreased as the leachate concentration increased. With the increase of incubation time, the HEK293 and 3T3 cells multiplied in all the leachates except $0.1 \%$ phenol (the positive control). Overall, the cytotoxicity of EGF-Cur-NP/H is sufficiently low on both HEK293 and 3T3 cells according to the good cell viability and proliferation.

\section{In vitro drug release profile}

As illustrated in Figure 4C and D, approximately 90\% of EGF and $60 \%$ of Cur were released within 24 hours from the free drug groups, while the EGF-Cur-NP and EGF-Cur-NP/H groups showed slow cumulative release profiles of EGF and Cur. Attributing to the internal structural characteristics of polymeric hydrogel, the release rate of the EGF-Cur-NP/H group was much slower relative to the EGF-Cur-NP group.
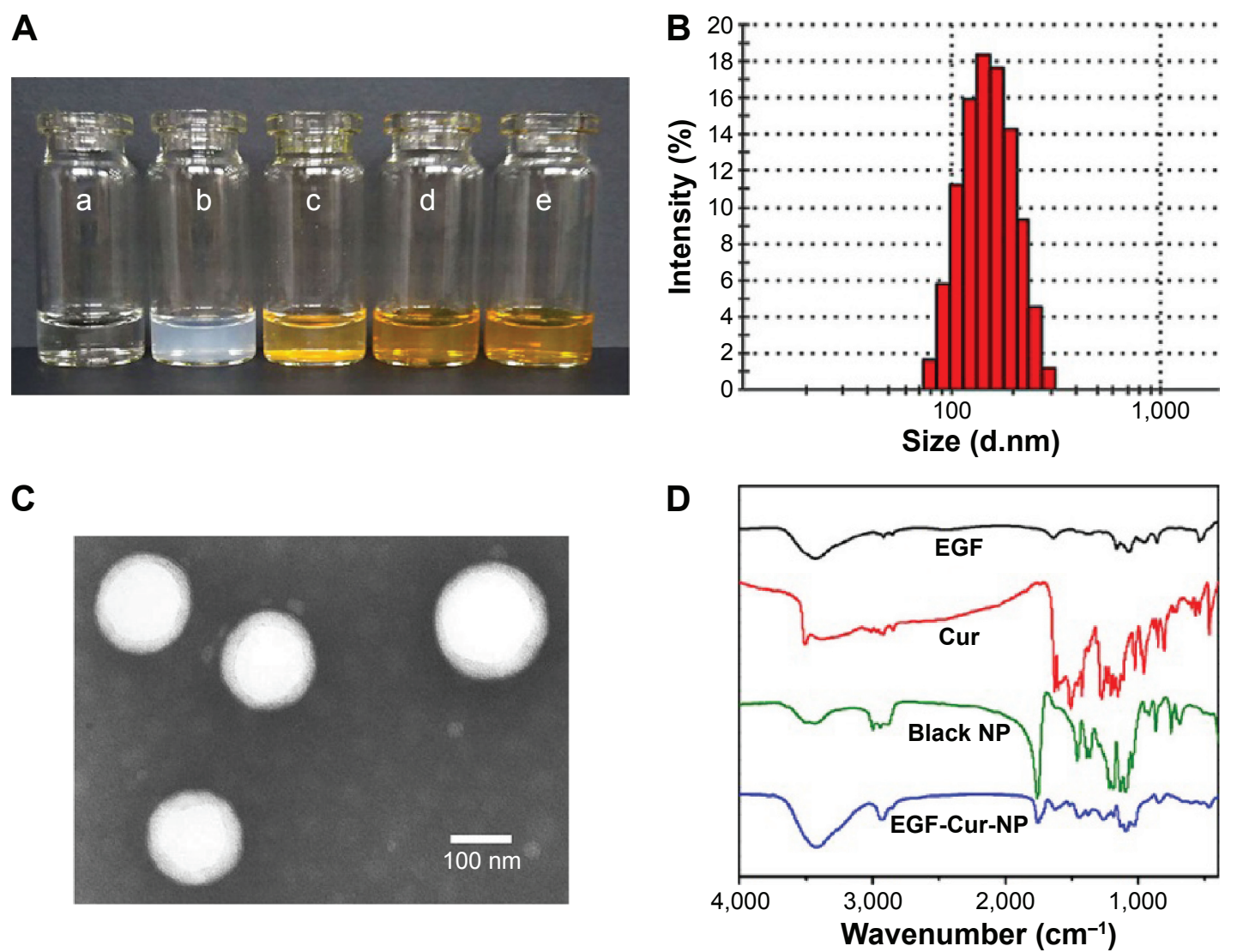

Figure 2 Characterization of PLA- IOR5-PLA copolymer NPs.

Notes: (A) Morphology of normal saline (NS) (a), EGF-NP (b), Cur in NS (c), Cur-NP (d), and EGF-Cur-NP (e); (B) particle size distribution of EGF-Cur-NP; (C) TEM image of EGF-Cur-NP (magnification 50,000×); (D) FTIR spectrum of EGF, Cur, the black NP, and EGF-Cur-NP.

Abbreviations: EGF, epidermal growth factor; NP, nanoparticle; Cur, curcumin; TEM, transmission electron microscopy; FTIR, Fourier transform infrared spectroscopy. 
Table 2 The PLA-IOR5-PLA copolymer nanoparticles prepared in this work

\begin{tabular}{lllllll}
\hline Code & Size & PDI & DL (\%) of Cur & DL (\%) of EGF & EE (\%) of Cur & EE (\%) of EGF \\
\hline SI & $123.4 \pm 3.8$ & $0.132 \pm 0.067$ & - & - & - & - \\
S2 & $135.3 \pm 3.2$ & $0.091 \pm 0.038$ & $9.35 \pm 0.21$ & - & $93.50 \pm 2.10$ & - \\
S3 & $131.3 \pm 4.5$ & $0.089 \pm 0.015$ & - & $4.33 \pm 0.21$ & - & $86.60 \pm 4.20$ \\
S4 & $145.3 \pm 2.8$ & $0.115 \pm 0.022$ & $8.75 \pm 0.32$ & $4.03 \pm 0.15$ & $91.91 \pm 3.36$ & $84.66 \pm 3.15$ \\
\hline
\end{tabular}

Notes: SI, the black nanoparticles; S2, curcumin loaded nanoparticles; S3, EGF loaded nanoparticles; S4, curcumin and EGF co-loaded nanoparticles. “-”, no data.

Abbreviations: PLA, polylactic acid; IOR5, reverse Pluronic ${ }^{\circledR}$ IOR5; Cur, curcumin; PDI, polydispersity index; DL, drug loading; EE, encapsulation efficiency; EGF, epidermal growth factor.

\section{In vivo wound healing assay}

Macroscopic observations of wound closure for all treatment groups at 3, 7, 10, and 14 days post-wounding are shown in Figure 5. Topical administration of EGF-Cur-NP/H significantly accelerated wound closure with no scabbing observed in contrast with the NS, nanoparticle/hydrogel $(\mathrm{NP} / \mathrm{H}), \mathrm{EGF}-\mathrm{NP} / \mathrm{H}$, and Cur-NP/H groups. At day 3 postinjury, the NS group showed signs of hemorrhage and inflammation in the wounds. Figure $6 \mathrm{~A}$ revealed that on the 14th postoperative day, the closure rate of the EGF-Cur-NP/H group $(94.78 \% \pm 3.12 \%)$ was significantly higher compared with the Cur-NP/H $(87.58 \% \pm 3.92 \%, P<0.05)$, EGF-NP/H
(86.47\% $\pm 4.62 \%, P<0.05), \mathrm{NP} / \mathrm{H}(72.96 \% \pm 4.26 \%, P<0.05)$, or NS groups $(65.96 \% \pm 4.93 \%, P<0.05)$.

H\&E staining of sections from day 7 and 14 revealed distinct differences in quality of granulation tissue and maturity of the epidermis/dermis among EGF-Cur-NP/H and the other groups (Figures 6B, and C). The EGF-NP/H, Cur-NP/H, and EGF-Cur-NP/H treated groups demonstrated enhanced healing response; on day 14 , accelerated wound maturation and a well formed epidermis with compact orthokeratosis were observed in these groups. However, much well-organized granulation tissue was exhibited in the EGF-Cur-NP/H group. Furthermore, the NS and NP/H treated wounds displayed

\section{A}
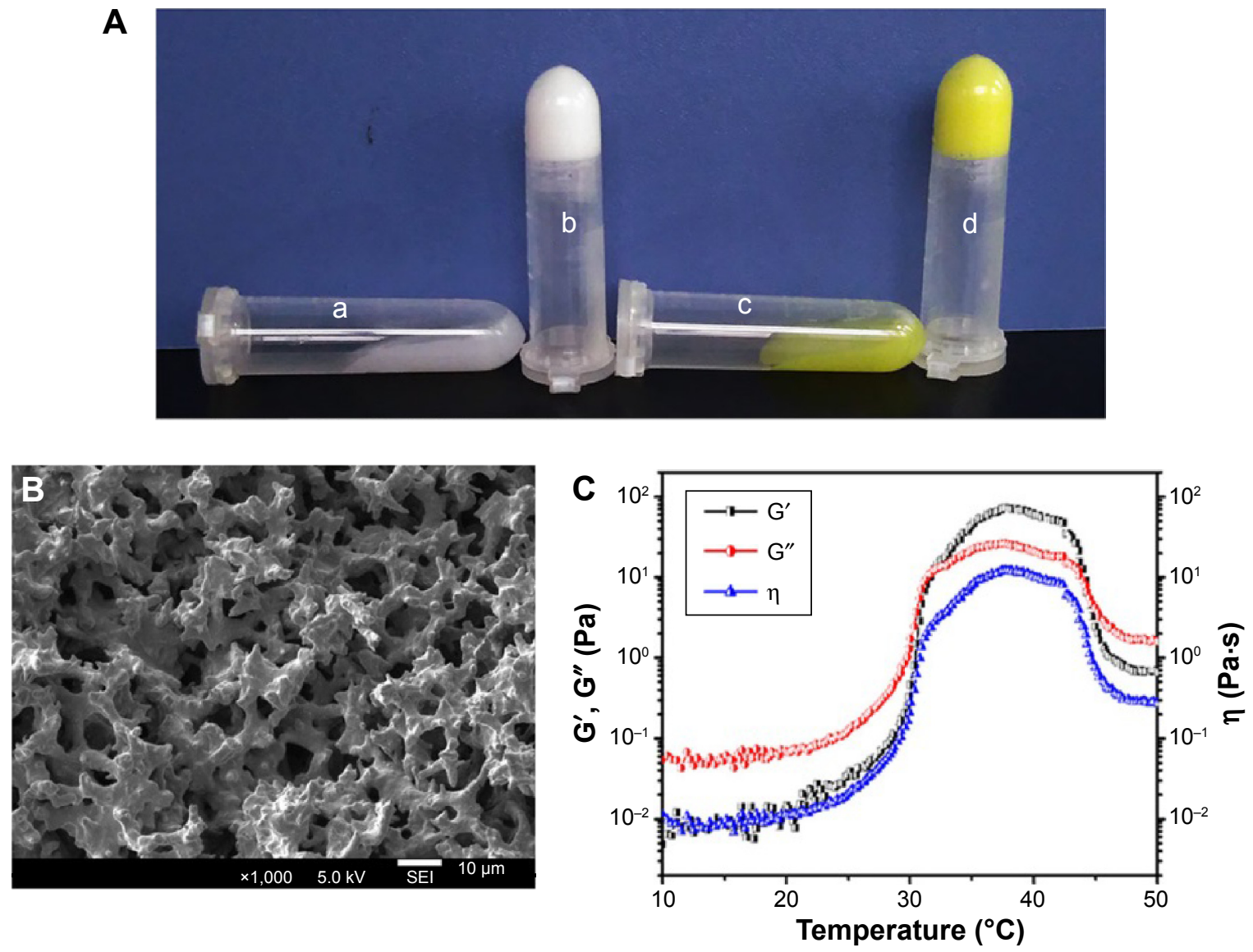

Figure 3 Characterization of EGF-Cur-NP/H

Notes: (A) Photographs of the black hydrogel (a), EGF-Cur-NP/H (c) exhibiting a free-flowing sol at $25^{\circ} \mathrm{C}$ and a solid-like gel after being heated to $37^{\circ} \mathrm{C}$ (b, d); (B) SEM image of EGF-Cur-NP/H; (C) rheology analysis of EGF-Cur-NP/H as a function of temperature.

Abbreviations: EGF, epidermal growth factor; NP, nanoparticle; H, hydrogel; Cur, curcumin; SEM, scanning electron microscopy; G', storage modulus; G", loss modulus; $\eta$, viscosity. 


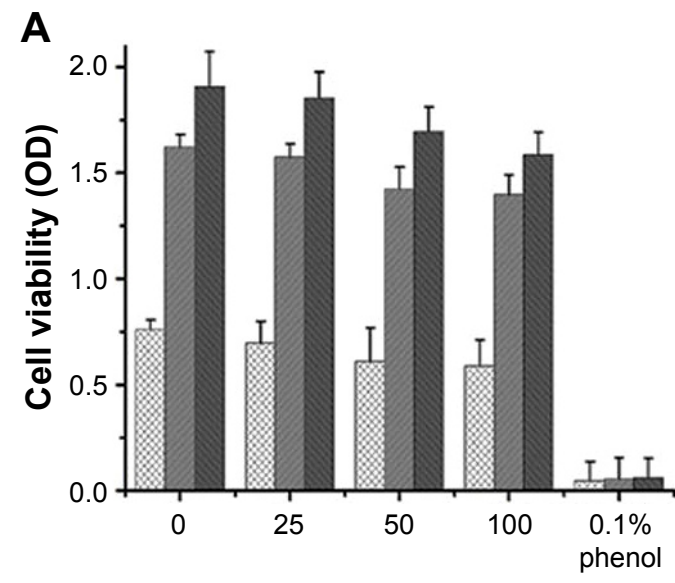

Leachate concentration (\%)

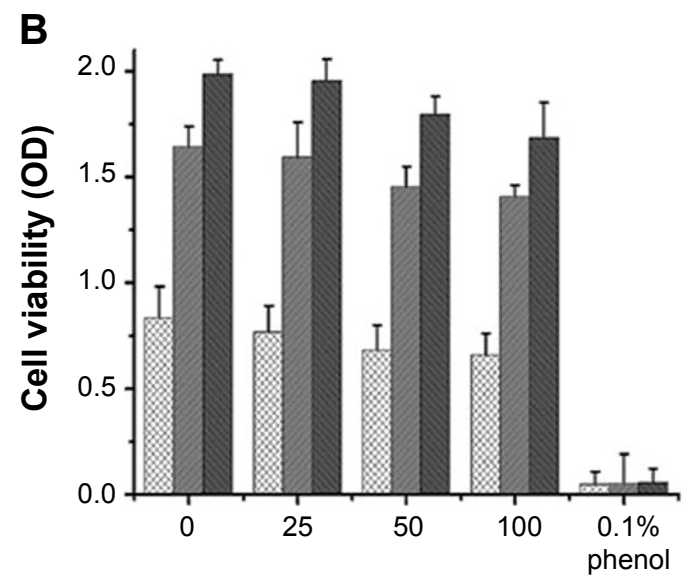

Leachate concentration (\%)

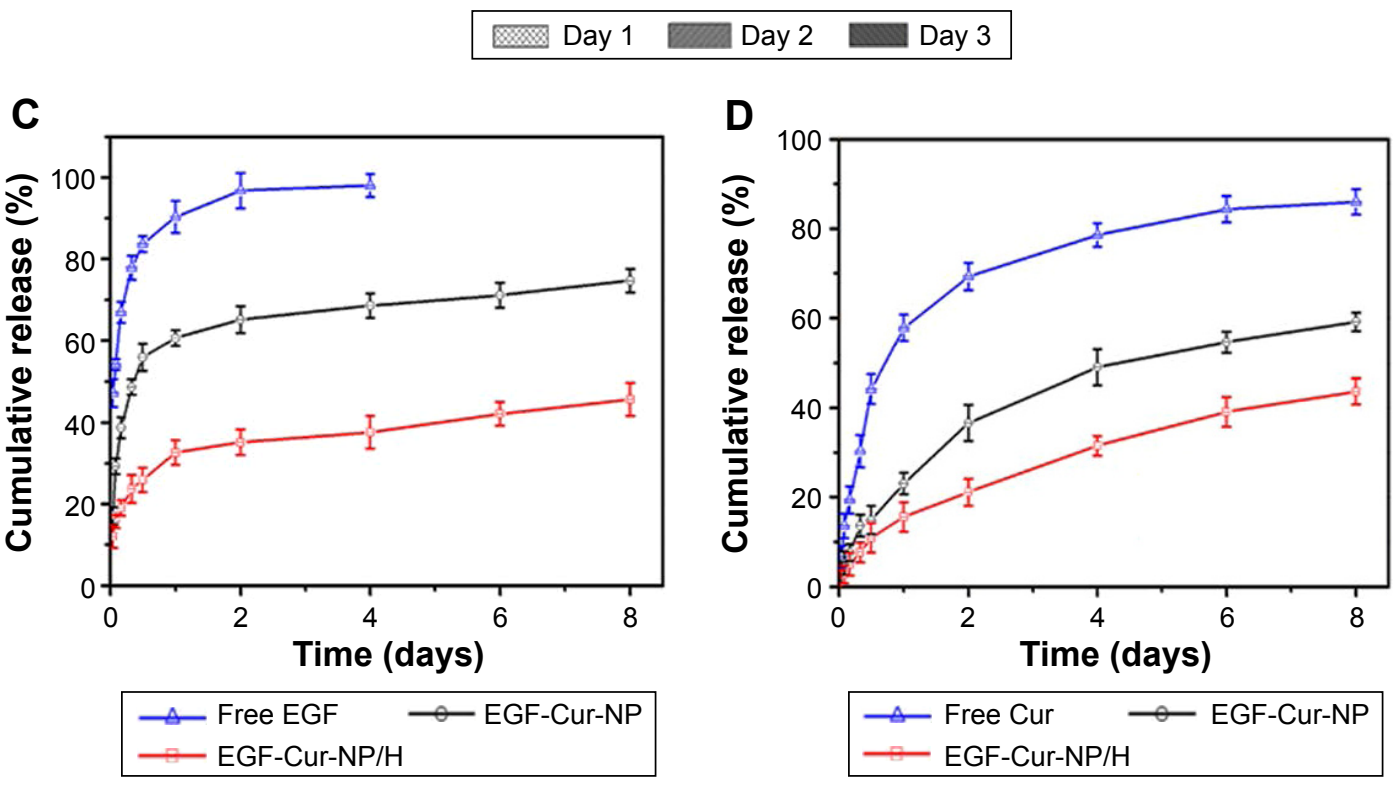

Figure 4 In vitro cytotoxicity and drug release study.

Notes: Cell viability test of HEK293 cells (A) and 3T3 cells (B) after incubation with EGF-Cur-NP/H extracts of different concentrations (I00\%, 50\%, 25\%, 0\% as the negative control, and $0.1 \%$ phenol as the positive control). Data were presented as mean $\pm S D, n=6$. In vitro drug release profile of EGF from free EGF, EGF-Cur-NP, and EGF-Cur-NP/H (C) and Cur from free Cur, EGF-Cur-NP, and EGF-Cur-NP/H (D) in PBS solution at $\mathrm{pH}$ 7.4. Data were presented as mean \pm SD, $n=3$.

Abbreviations: EGF, epidermal growth factor; OD, optical density; NP, nanoparticle; H, hydrogel; Cur, curcumin; SD, standard deviation; PBS, phosphate-buffered saline.

more inflammatory granulation tissue and incomplete reepithelialization of the epidermis with overlying serum crust until the 14th day post-wounding.

MT staining was used to evaluate collagen deposition. As illustrated in Figure 6D and Figure 7, the EGF-Cur-NP/H group showed a higher extent of collagen deposition and more compact and denser collagen alignment through the whole wound healing process as compared with the other groups. It was observed that the wounds treated with NS, $\mathrm{NP} / \mathrm{H}$, EGF-NP/H, or Cur-NP/H contained a subjectively greater degree of mixed inflammatory infiltrate splaying between the collagen on day 14. Moreover, semi-quantitative analysis of collagen revealed significantly increased collagen intensity in EGF-Cur-NP/H treated wounds (68.25\% $\pm 7.49 \%$ on day $7,85.68 \% \pm 4.54 \%$ on day 14$)$ than the aforementioned controls $(P<0.05, \mathrm{n}=6)$.

Figure 8 suggested that the Cur-NP/H and EGF-Cur$\mathrm{NP} / \mathrm{H}$ treated groups exhibited greater intensity of staining for TGF- $\beta 1$, while only a faint staining was seen in the other groups. Immunofluorescence staining of CD31 (Figure 9) revealed significantly greater neovascularization in EGFCur-NP/H treated group compared with all other groups, which was evaluated by number of stained microvessels per high-power field $(20 \times, P<0.05, \mathrm{n}=10)$.

\section{Discussion}

Optimum healing of cutaneous wounds requires a complex physiological response process involving four distinct, but 


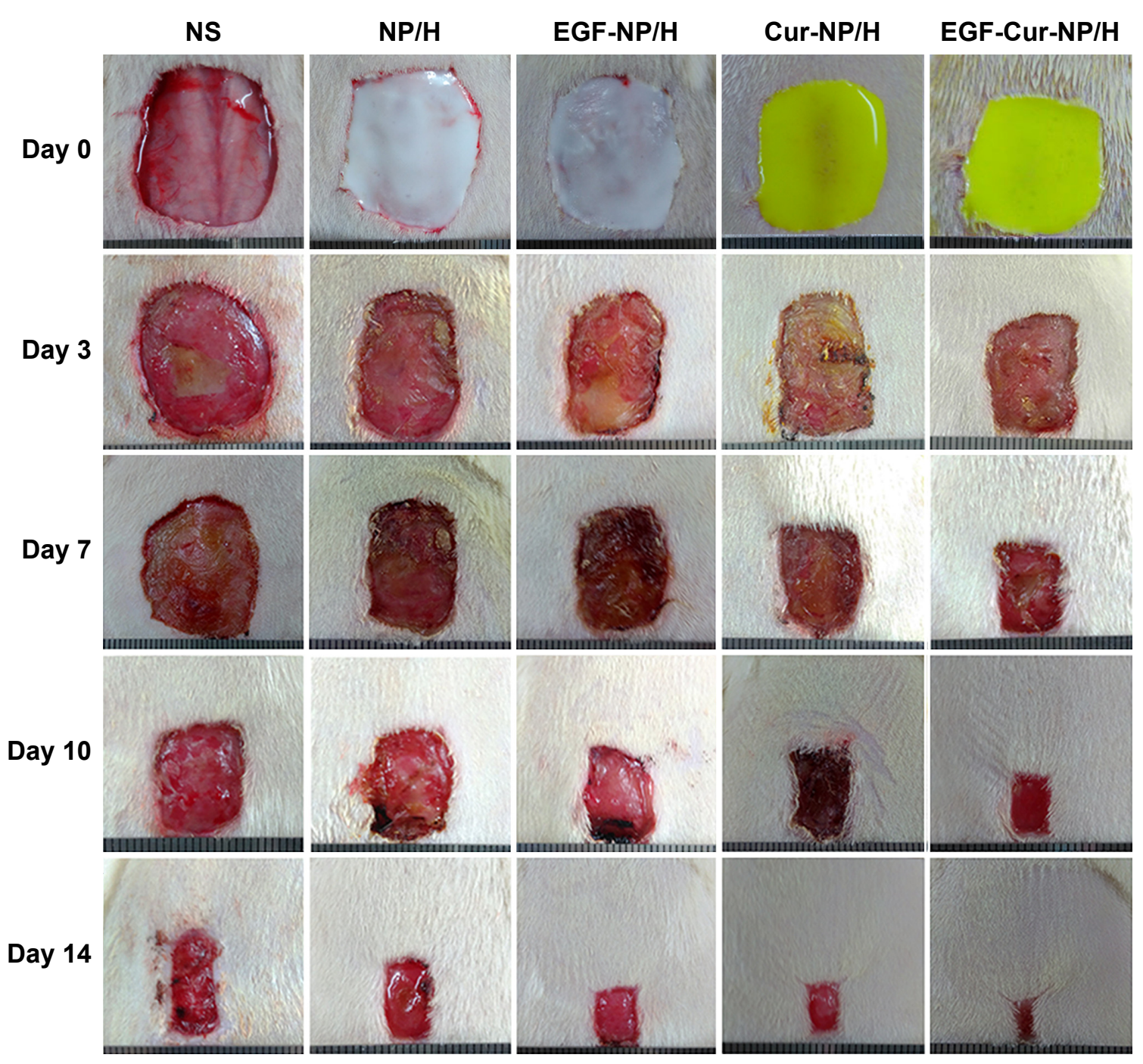

Figure 5 Macroscopic observations of wound closure for NS, NP/H, EGF-NP/H, Cur-NP/H, and EGF-Cur-NP/H treated groups at day 0, 3, 7, I0, and I4 post-wounding. Abbreviations: EGF, epidermal growth factor; NS, normal saline; NP, nanoparticle; $\mathrm{H}$, hydrogel; Cur, curcumin.

overlapping phases: hemostasis, inflammation, proliferation, and remodeling. ${ }^{3,27}$ Efficient wound repair is essential to protect the body against debris and foreign pathogens, while any disturbances in the process may impair the restoring of tissue architecture and function, resulting in chronic wounds or even uncontrolled wound healing. . $^{28,29}$ There has been little improvement in preventing morbidity and disability from chronic wounds over the past decades. ${ }^{30}$ Therefore, it is imperative to cover the injury with an effective dressing as early as possible to remove exudate, inhibit exogenous microorganism invasion, and initiate rapid skin regeneration. ${ }^{31,32}$

It is widely accepted that a warm, moist wound healing environment could optimize the healing rates, and various wound care products such as hydrogels, films, sponges, and nanofibers are designed to provide these conditions. ${ }^{33,34}$ The biocompatible hydrogels with in situ gel-forming properties have aroused a great interest in constructing wound dressing owing to many of their desirable advantages such as provision of a moist environment, flexibility of loading growth factors or drugs, proper adherence without wrinkling or fluting in the wound bed, ease of application, and improved patient compliance. ${ }^{35-37}$

In the present study, the PLA-10R5-PLA copolymer with a molecular mass of $4.6 \times 10^{3}$ was administered to fabricate the thermosensitive hydrogel. In addition, the aqueous sol could be easily mixed with drug-loaded NPs to formulate an in situ gel forming system for certain applications. As shown in Figure 3, the copolymer aqueous solution is a free-flowing sol at room temperature, but forms a solid-like gel when heated to $37^{\circ} \mathrm{C}$ without any cross-linker or UV illumination. The aqueous solution of 10R5 (30 wt \%) did not show sol-gel transition in a temperature range of $10^{\circ} \mathrm{C}-60^{\circ} \mathrm{C}$ mainly due to its high $\mathrm{CMC}$, which is validated by the results of rheology 
A

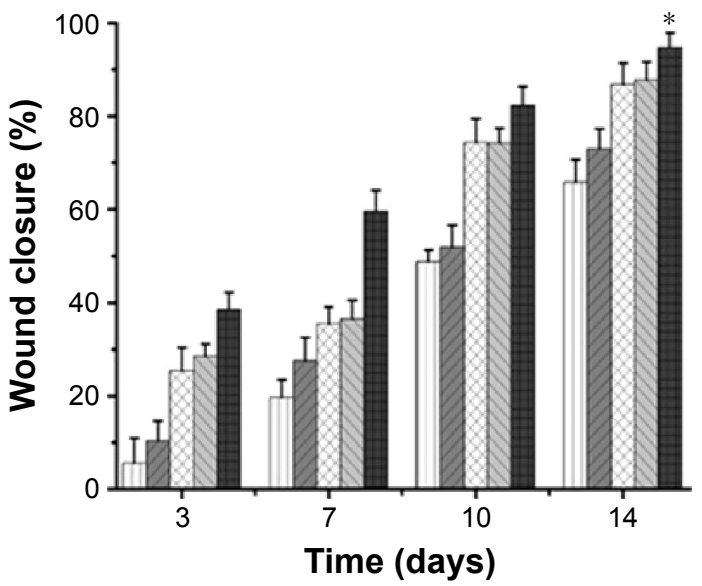

B

$$
\text { Day } 7 \quad \square Z \square \text { Day } 14
$$
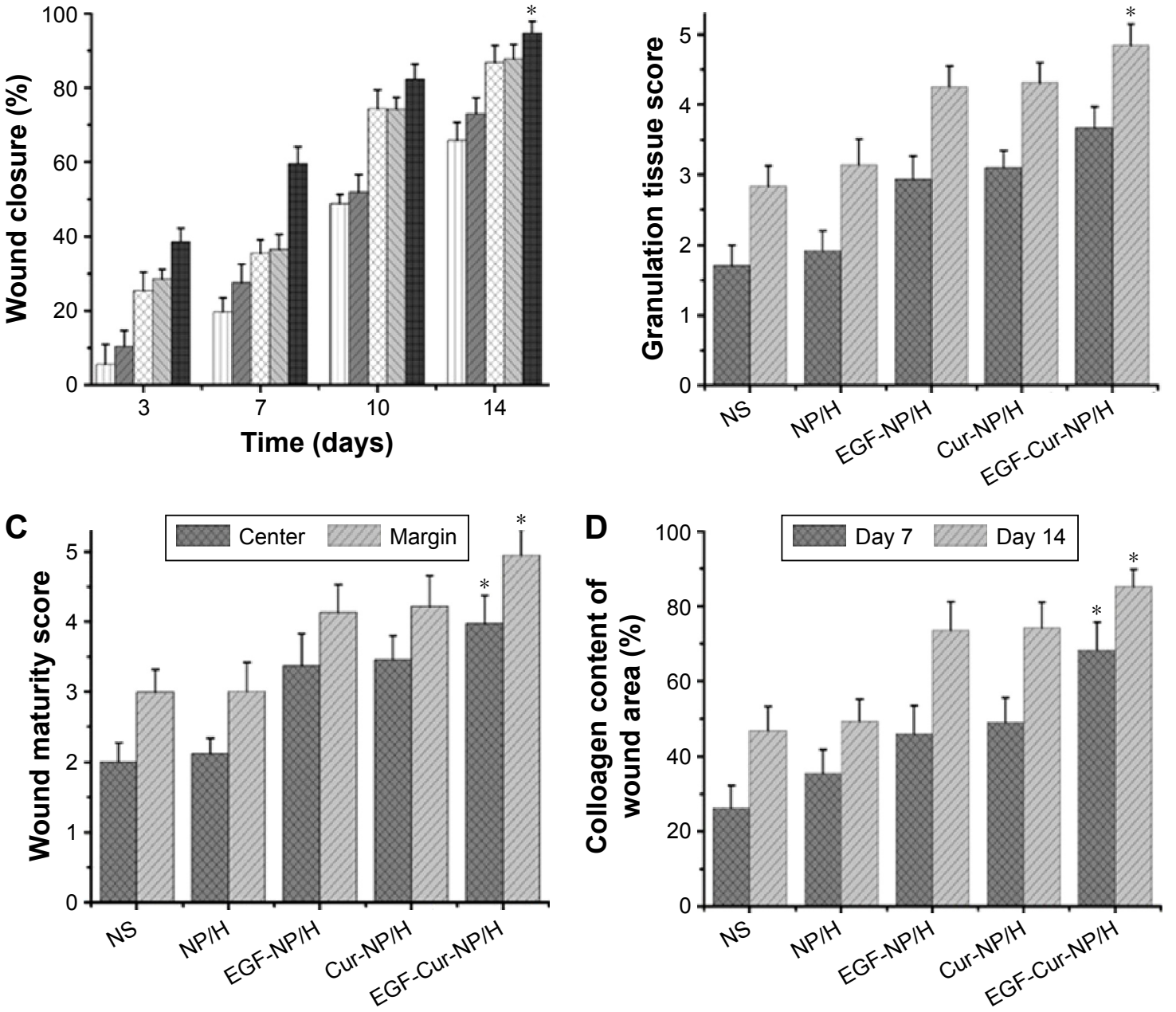

Figure 6 In vivo wound healing effects of EGF-Cur-NP/H.

Notes: (A) Wound closure rate of all the groups at day 3, 7, 10, and 14; (B) the granulation tissue formation score of all the groups at day 7 and I4; (C) the wound maturity score of all the groups at day I4; (D) semi-quantitative analysis of collagen deposition at day 7 and I4. Data were presented as mean \pm SD, $n=6$, $* P<0.05$ was considered significant.

Abbreviations: EGF, epidermal growth factor; NP, nanoparticle; H, hydrogel; Cur, curcumin; SD, standard deviation; NS, normal saline.

analysis shown in Figure S4. Moreover, the NP/hydrogel composite (EGF-Cur-NP/H) also showed sol-gel transition in a certain temperature range at approximately $37^{\circ} \mathrm{C}$, and MTT assay (Figure 4A and B) suggests that EGF-Cur-NP/H is biocompatible with quite low cytotoxicity on HEK293 and 3T3 cells, which indicated the potential application of the copolymer hydrogel in wound management.

Numerous studies have documented that topical use of EGF, an extensively investigated growth factor, could accelerate epidermal and mesenchymal regeneration, as well as cell motility and proliferation. ${ }^{6,9,14,38}$ Many efforts have recently been devoted to develop efficient delivery systems for EGF topical application in wound repair. ${ }^{16,39,40}$
To facilitate the application, some growth factors were incorporated directly into hydrogel-like formulations, but the active compounds exhibit a rapid release profile from these formulations. ${ }^{41}$ Though novel drug delivery systems like microspheres, NPs, and liposomes could prolong the release profile of the proteins, the topical administration of these drug carrier vehicles gave rise to a rapid leakage of the molecules from the wound site. ${ }^{42}$ Thus, we hypothesized that encapsulation of EGF into an in situ gel-forming NP-hydrogel system may optimize its topical administration in terms of dose, delivery pattern, and safety.

Cur promotes wound healing by decreasing the release of inflammatory cytokines, increasing formation of 
A

Day 7

Day 14

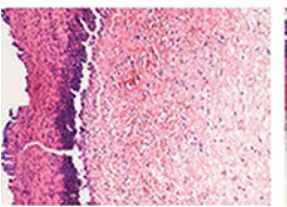

B

Day 7

Day 14

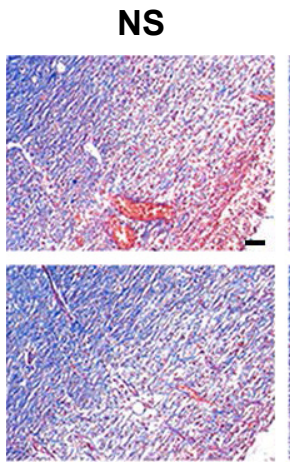

NP/H
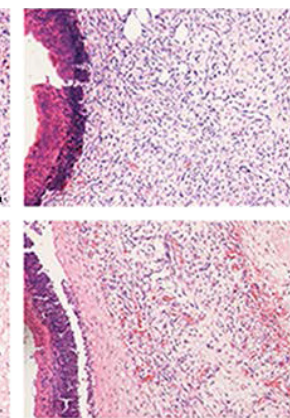

NP/H

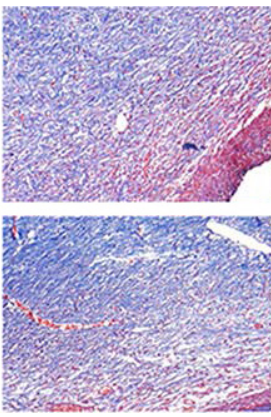

EGF-NP/H
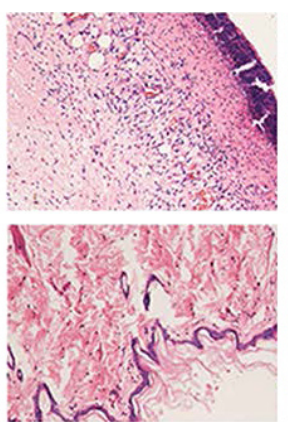

EGF-NP/H
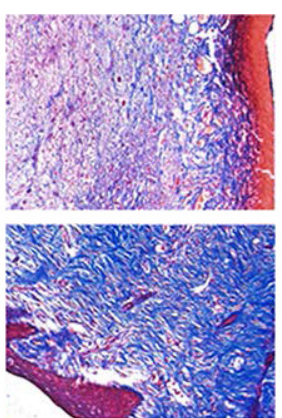

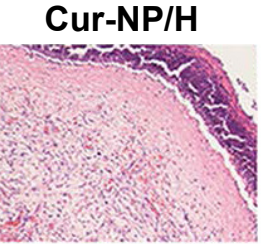

EGF-Cur-NP/H
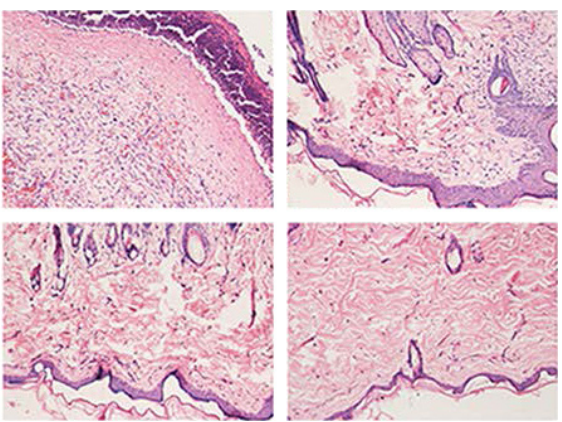

Cur-NP/H

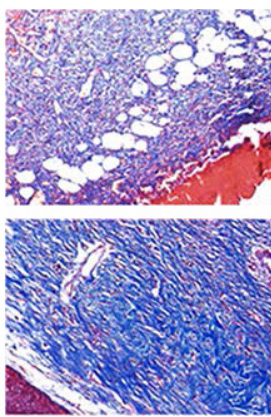

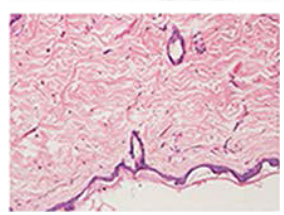

EGF-Cur-NP/H

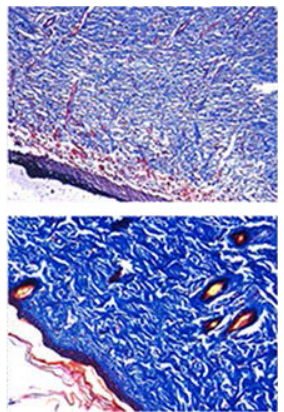

Figure $7 \mathrm{H \& E}$ and $M T$ staining of the wounds.

Notes: Representative images of H\&E (A), and MT (B) staining of wounds treated with NS, NP/H, EGF-NP/H, Cur-NP/H, and EGF-Cur-NP/H at day 7 and 14 post-wounding. Scale bar $=50 \mu \mathrm{m}$, magnification $20 \times$

Abbreviations: EGF, epidermal growth factor; H\&E, hematoxylin and eosin; MT, Masson's trichrome; NP, nanoparticle; H, hydrogel; Cur, curcumin; NS, normal saline.
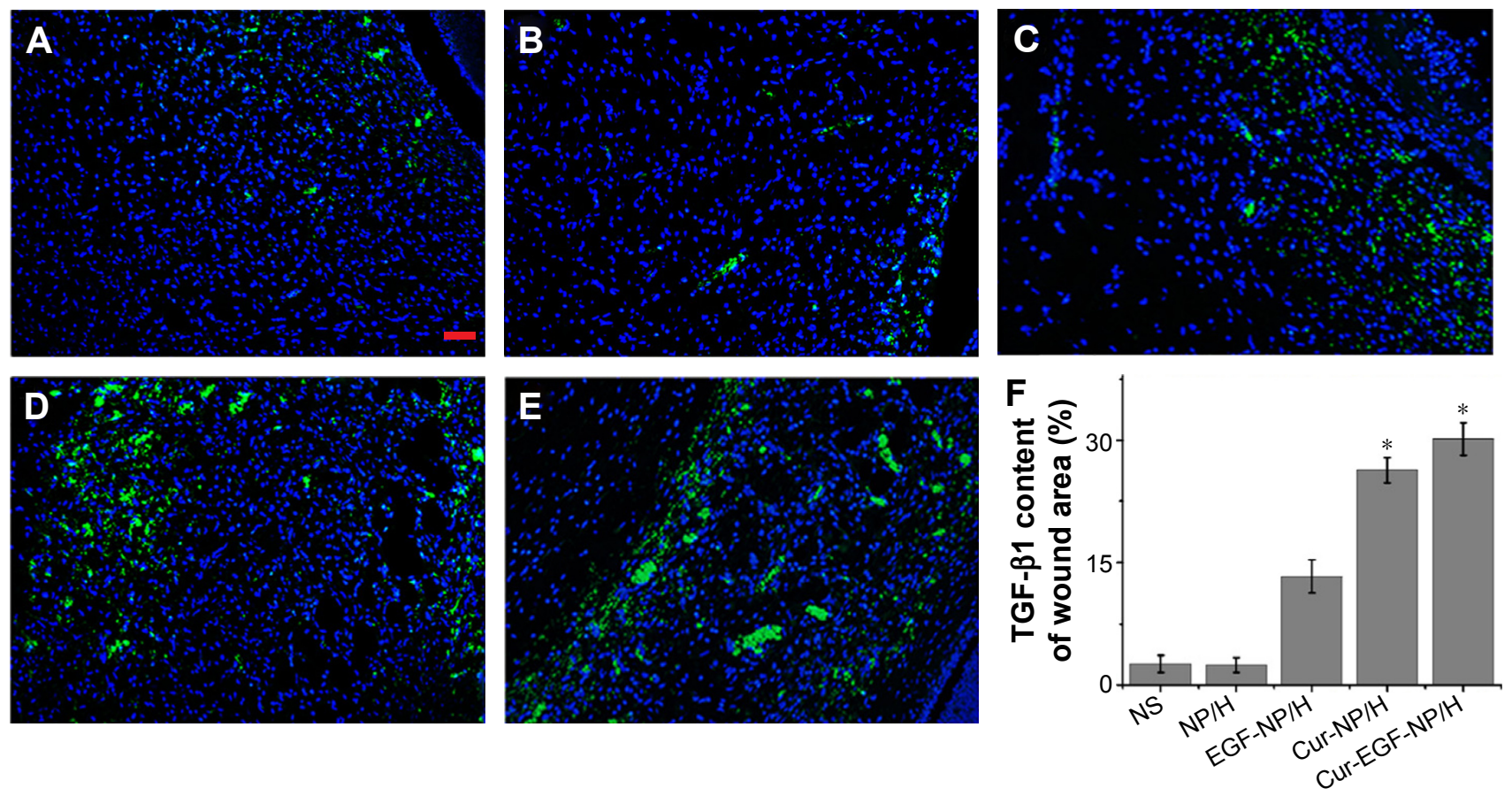

Figure 8 TGF- $\beta$ I staining of the wounds.

Notes: Fluorescent microscopy of TGF- $\beta$ I staining wounds treated with NS (A), NP/H (B), EGF-NP/H (C), Cur-NP/H (D), and EGF-Cur-NP/H (E) at day 7 post-wounding. Nuclei were stained blue with DAPI, and TGF- $\beta$ I was stained green. (F) Semi-quantitative estimation of TGF- $\beta$ I expression in each group. Data were presented as mean $\pm S D, n=10, * P<0.05$ was considered significant. Scale bar $=50 \mu \mathrm{m}$, magnification $20 \times$.

Abbreviations: NS, normal saline; NP, nanoparticle; H, hydrogel; EGF, epidermal growth factor; Cur, curcumin; DAPI, 4',6-diamidino-2-phenylindole; SD, standard deviation; TGF- $\beta$ I, transforming growth factor beta-I. 

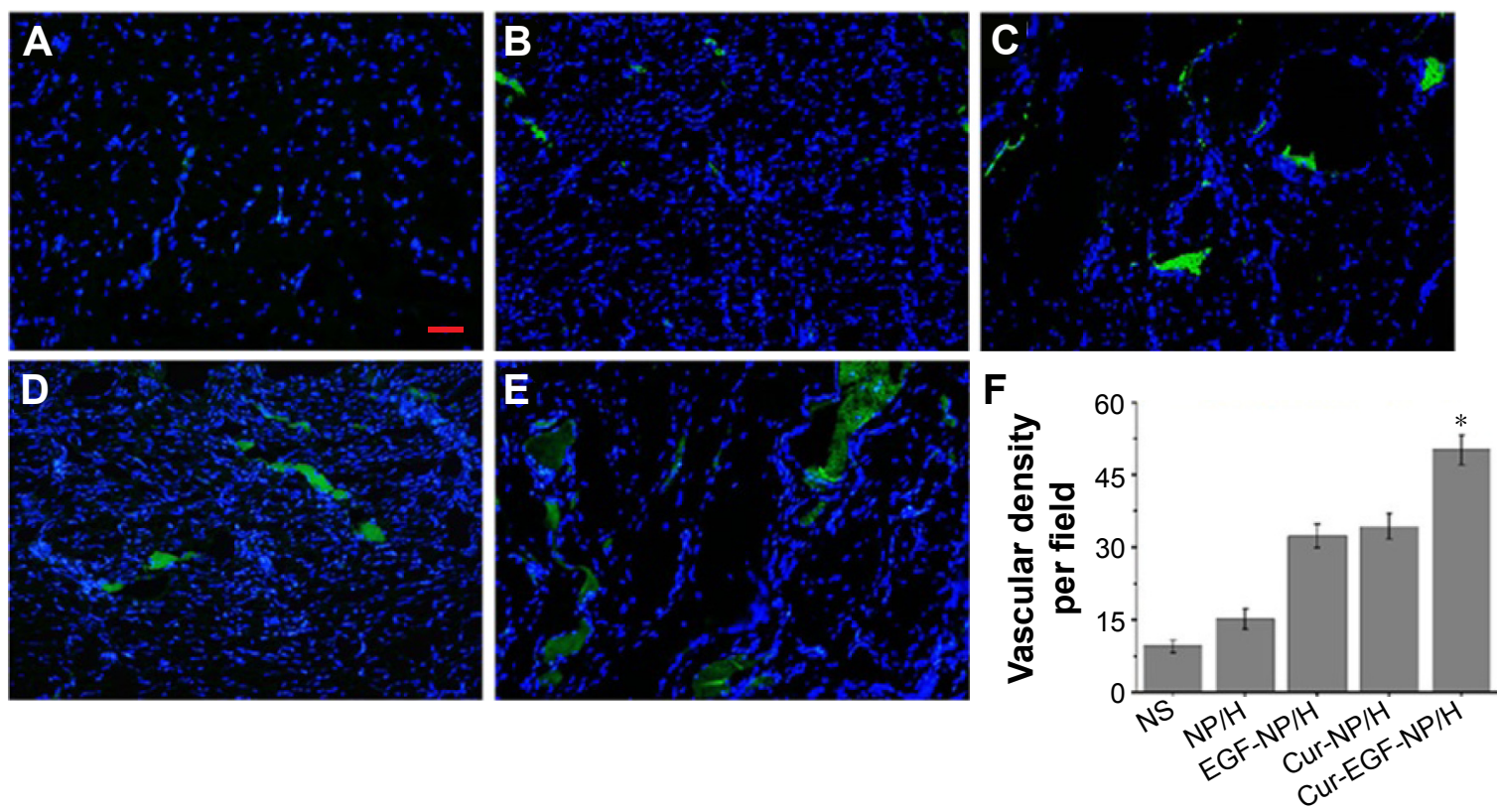

Figure $9 \mathrm{CD} 3 \mathrm{I}$ staining of the wounds.

Notes: Fluorescent microscopy of CD3I staining wounds treated with NS (A), NP/H (B), EGF-NP/H (C), Cur-NP/H (D), and EGF-Cur-NP/H (E) at day I4 post-wounding. Nuclei were stained blue with DAPI, and the newly formed vessels were stained green. (F) Vascular density of each group. Data were presented as mean $\pm S D, n=10, * P<0.05$ was considered significant. Scale bar $=50 \mu \mathrm{m}$, magnification $20 \times$.

Abbreviations: NS, normal saline; NP, nanoparticle; H, hydrogel; EGF, epidermal growth factor; Cur, curcumin; DAPI, 4',6-diamidino-2-phenylindole; SD, standard deviation.

granulation tissue and neovascularization, and enhancing the biosynthesis of TGF- $\beta 1$ and ECM proteins, such as collagen. ${ }^{43-45}$ TGF- $\beta 1$ is important in inflammation, angiogenesis, re-epithelialization, and accumulation of ECM. ${ }^{46}$ Furthermore, it induces down-regulation of NFKB pathway to inhibit the oxidative damage in human keratinocytes and fibroblasts. ${ }^{47}$ However, the hydrophobicity of Cur has limited its use in clinical practice. ${ }^{48}$ And nanotechnology has been widely used to improve the water solubility of hydrophobic drugs through encapsulation in polymer micelles, NPs, and liposomes. ${ }^{49}$ To achieve a much accelerated wound contract rate, EGF and Cur were co-loaded in the copolymer NPs by w/o/w emulsion solvent evaporation method, and then EGFCur-NPs were dispersed in the PLA-10R5-PLA hydrogel at room temperature and used as dual drug loaded wound dressing.

The prepared EGF-Cur-NP is mono-dispersed with a particle size of $145.3 \pm 2.8 \mathrm{~nm}$ and a PDI of $0.115 \pm 0.022$ (Figure 2 and Table 2). Considering the chemical structure of PLA-10R5-PLA (PLA-PPG-PEG-PPG-PLA), the watersoluble peptide EGF may combine with the PEG segments as the hydrophilic shells of the particles, while Cur with quite low aqueous solubility may be loaded in the hydrophobic core formed by the PPG and PLA blocks. The in vitro drug release profiles shown in Figure 4C and D indicated that EGF and Cur were released in a sustained manner when encapsulated in the copolymer NP. And due to the interaction of the hydrogel structure, the agents were released much slower from EGF-Cur-NP/H, which can act as both the wound dressing and a sustained drug depot.

The wound healing capabilities of EGF-Cur-NP/H were evaluated in a rat full thickness excisional model. As shown in Figure 5, EGF-Cur-NP/H exhibited much faster recovery of the wounds compared to that of only EGF- or Cur-NP/H, which attributed to the combined effects of EGF and Cur. In addition to accelerated closure, histological examination of H\&E, and MT stained sections demonstrated that EGF-Cur-NP/H treated wounds displayed much faster wound maturation and re-epithelialization, more well-formed granulation tissue, as well as more compact and denser collagen alignment. Furthermore, immunofluorescence assay showed that Cur could enhance the biosynthesis of TGF- $\beta 1$. CD31 staining exhibited enhanced new vessel formation in the wounds treated with EGF-Cur-NP/H. It is already known that angiogenesis is critical to the process of wound healing because it enables the supply of oxygen and nutrients to the newly formed granulation tissue and the keratinocytes. ${ }^{50}$

In summary, the copolymer NP/hydrogel system presents a significant advance in the co-encapsulation of EGF and Cur, which circumvents the difficulties inherent in EGF and Cur topical administration, enabling sustained delivery of 


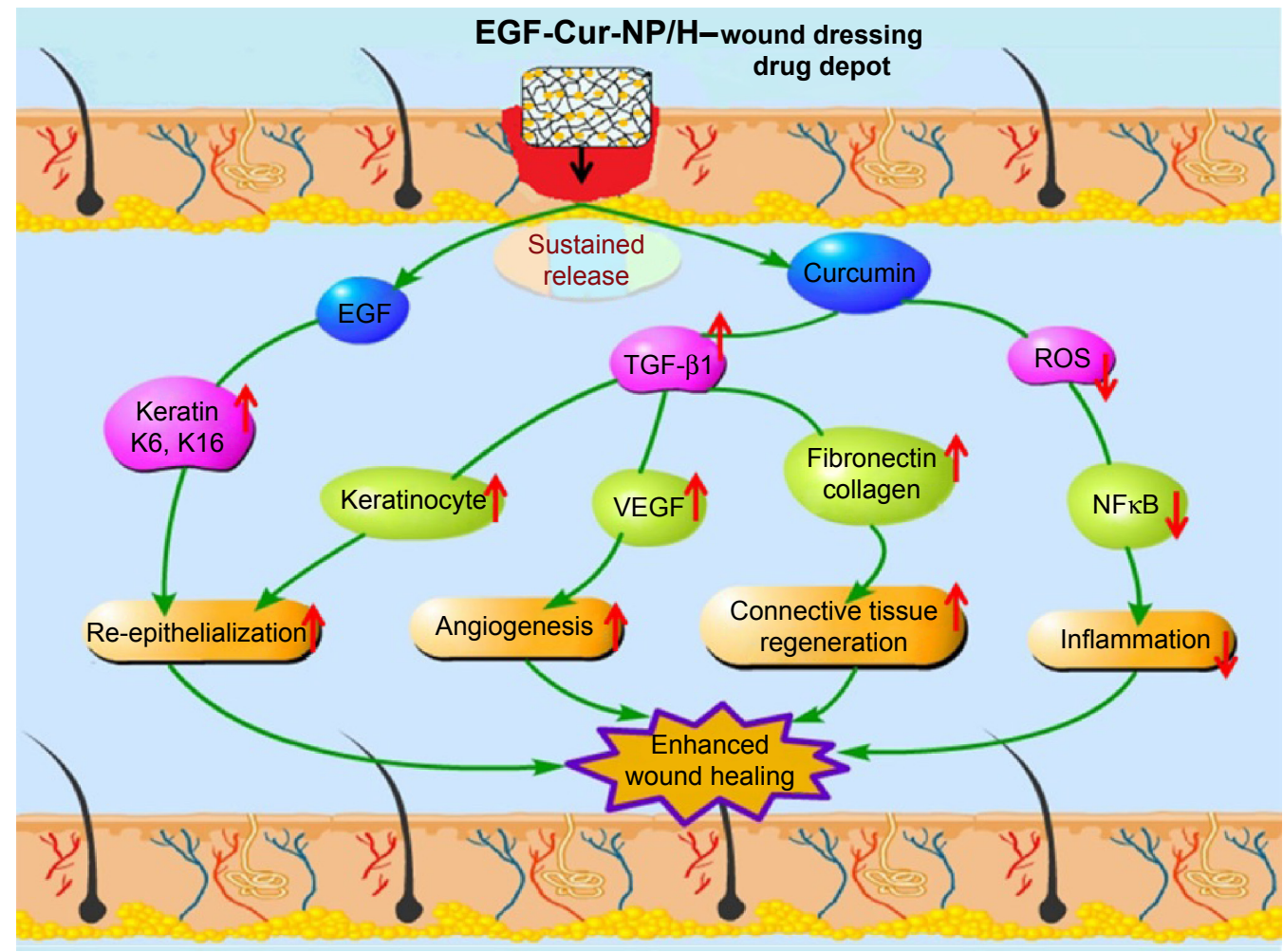

Figure 10 Schematic representation of mechanisms of action of Cur and EGF in wound healing processes.

Abbreviations: NP, nanoparticle; H, hydrogel; Cur, curcumin; EGF, epidermal growth factor; ROS, reactive oxygen species; TGF- $\beta$ I, transforming growth factor beta-I.

these therapeutic agents. Unlike current treatment options, EGF-Cur-NP/H decreases the risk of infections and significantly enhances wound healing rate due to the suitable environment provided by the thermosensitive hydrogel and the combined wound repair effects of EGF and Cur. Thus, EGF-Cur-NP/H has the potential to serve as a novel topical agent for cutaneous wound healing.

\section{Conclusion}

In this study, we reported the development of a novel in situ gel-forming controlled dual-drug delivery system (EGF-Cur-NP/H) and its therapeutic activities to accelerate dermal wound healing were investigated in a full-thickness excision wound model. EGF-Cur-NP/H exhibited rather low cytotoxicity and sustained drug release behavior in vitro. Furthermore, it was an injectable sol at room temperature, and after application to the wound, it became a non-flowing gel. EGF-Cur-NP/H showed excellent wound healing activity in vivo through increasing granulation tissue formation, collagen deposition, and angiogenesis compared with EGF-NP/H or Cur-NP/H (Figure 10). Due to its effectiveness and safety, the biodegradable and injectable EGF-Cur-NP/H is a promising candidate as wound dressing.

\section{Acknowledgments}

This work was financially supported by National Natural Science Foundation of China (31471286), National S\&T Major Project (2015ZX09102010), and Sichuan Provincial Science and Technology Department Support Project (2011SZ0222).

\section{Disclosure}

The authors report no conflicts of interest in this work.

\section{References}

1. Zielins ER, Atashroo DA, Maan ZN, et al. Wound healing: an update. Regen Med. 2014;9(6):817-830.

2. Singer AJ, Clark RA. Cutaneous wound healing. N Engl J Med. 1999; 341(10):738-746.

3. Gurtner GC, Werner S, Barrandon Y, Longaker MT. Wound repair and regeneration. Nature. 2008;453(7193):314-321.

4. Tang T, Jiang H, Yu Y, et al. A new method of wound treatment: targeted therapy of skin wounds with reactive oxygen species-responsive nanoparticles containing sDF-1 $\alpha$. Int $J$ Nanomedicine. 2015;10: 6571-6585.

5. Sun BK, Siprashvili Z, Khavari PA. Advances in skin grafting and treatment of cutaneous wounds. Science. 2014;346(6212):941-945.

6. Werner S, Grose R. Regulation of wound healing by growth factors and cytokines. Physiol Rev. 2003;83(3):835-870.

7. Barrientos S, Stojadinovic O, Golinko MS, Brem H, Tomic-Canic M. Growth factors and cytokines in wound healing. Wound Repair Regen. 2008;16(5):585-601. 
8. Choi JK, Jang JH, Jang WH, et al. The effect of epidermal growth factor (EGF) conjugated with low-molecular-weight protamine (LMWP) on wound healing of the skin. Biomaterials. 2012;33(33): 8579-8590.

9. Johnson NR, Wang Y. Controlled delivery of heparin-binding EGF-like growth factor yields fast and comprehensive wound healing. $J$ Control Release. 2013;166(2):124-129.

10. Bae I, Park JW, Kim DY. Enhanced regenerative healing efficacy of a highly skin-permeable growth factor nanocomplex in a fullthickness excisional mouse wound model. Int J Nanomedicine. 2014;9: 4551-4567.

11. Xie Z, Paras CB, Weng H, et al. Dual growth factor releasing multifunctional nanofibers for wound healing. Acta Biomater. 2013;9(12): 9351-9359.

12. Chen RR, Mooney DJ. Polymeric growth factor delivery strategies for tissue engineering. Pharm Res. 2003;20(8):1103-1112.

13. Lehár J, Krueger AS, Avery W, et al. Synergistic drug combinations tend to improve therapeutically relevant selectivity. Nat Biotechnol. 2009;27(7):659-666.

14. Değim Z, Çelebi N, Alemdaroğlu C, et al. Evaluation of chitosan gel containing liposome-loaded epidermal growth factor on burn wound healing. Int Wound J. 2011;8(4):343-354.

15. Hardwicke J, Schmaljohann D, Boyce D, Thomas D. Epidermal growth factor therapy and wound healing - past, present and future perspectives. Surgeon. 2008;6(3):172-177.

16. Gorouhi F, Shah NM, Raghunathan VK, et al. Epidermal growth factorfunctionalized polymeric multilayer films: interplay between spatial location and bioavailability of EGF. J Invest Dermatol. 2014;134(6): $1757-1760$

17. Goel A, Kunnumakkara AB, Aggarwal BB. Curcumin as "Curecumin": from kitchen to clinic. Biochem Pharmacol. 2008;75(4):787-809.

18. Akbik D, Ghadiri M, Chrzanowski W, Rohanizadeh R. Curcumin as a wound healing agent. Life Sci. 2014;116(1):1-7.

19. Bhagavathula N, Warner RL, DaSilva M, et al. A combination of curcumin and ginger extract improves abrasion wound healing in corticosteroidimpaired hairless rat skin. Wound Repair Regen. 2009;17(3):360-366.

20. Krausz AE, Adler BL, Cabral V, et al. Curcumin-encapsulated nanoparticles as innovative antimicrobial and wound healing agent. Nanomedicine. 2015;11(1):195-206.

21. Guiraud S, Alimi-Guez D, van Wittenberghe L, Scherman D, Kichler A. The reverse block copolymer Pluronic 25R2 promotes DNA transfection of skeletal muscle. Macromol Biosci. 2011;11(5):590-594.

22. Naskar B, Ghosh S, Moulik SP. Solution behavior of normal and reverse triblock copolymers (Pluronic L44 and 10R5) individually and in binary mixture. Langmuir. 2012;28(18):7134-7146.

23. Hassanzadeh S, Feng Z, Pettersson T, Hakkarainen M. A proof-ofconcept for folate-conjugated and quercetin-anchored pluronic mixed micelles as molecularly modulated polymeric carriers for doxorubicin. Polymer. 2015;74:193-204.

24. Li X, Fan R, Wang Y, et al. In situ gel-forming dual drug delivery system for synergistic combination therapy of colorectal peritoneal carcinomatosis. RSC Advances. 2015;5(123):101494-101506.

25. Fan R, Tong A, Li X, et al. Enhanced antitumor effects by docetaxel/ 1137-loaded thermosensitive hydrogel nanoparticles in peritoneal carcinomatosis of colorectal cancer. Int J Nanomedicine. 2015;10: 7291-7305.

26. Hardwicke JT, Hart J, Bell A, et al. The effect of dextrin-rhEGF on the healing of full-thickness, excisional wounds in the $(\mathrm{db} / \mathrm{db})$ diabetic mouse. J Control Release. 2011;152(3):411-417.

27. Zeybel M, Hardy T, Wong YK, et al. Multigenerational epigenetic adaptation of the hepatic wound-healing response. Nat Med. 2012;18(9): 1369-1377.

28. Branski LK, Gauglitz GG, Herndon DN, Jeschke MG. A review of gene and stem cell therapy in cutaneous wound healing. Burns. 2009;35(2): 171-180.

29. Nauta A, Seidel C, Deveza L, et al. Adipose-derived stromal cells overexpressing vascular endothelial growth factor accelerate mouse excisional wound healing. Mol Ther. 2013;21(2):445-455.
30. Boulton AJM, Vileikyte L, Ragnarson-Tennvall G, Apelqvist J. The global burden of diabetic foot disease. Lancet. 2005;366(9498): 1719-1724.

31. Mohandas A, Sudheesh Kumar PT, Raja B, Lakshmanan VK, Jayakumar R. Exploration of alginate hydrogel/nano zinc oxide composite bandages for infected wounds. Int J Nanomedicine. 2015;10 Suppl 1: 53-66.

32. Jannesari M, Varshosaz J, Morshed M, Zamani M. Composite poly(vinyl alcohol)/poly(vinyl acetate) electrospun nanofibrous mats as a novel wound dressing matrix for controlled release of drugs. Int J Nanomedicine. 2011;6:993-1003.

33. Winter GD. Formation of the scab and the rate of epithelization of superficial wounds in the skin of the young domestic pig. Nature. 1962; 193:293-294.

34. MaverT,Hribernik S, Mohan T, Smrke DM, MaverU, Stana-KleinschekK. Functional wound dressing materials with highly tunable drug release properties. RSC Advances. 2015;5(95):77873-77884.

35. Murakami K, Aoki H, Nakamura S, et al. Hydrogel blends of chitin/ chitosan, fucoidan and alginate as healing-impaired wound dressings. Biomaterials. 2010;31(1):83-90.

36. Tran NQ, Joung YK, Lih E, Park KD. In situ forming and rutinreleasing chitosan hydrogels as injectable dressings for dermal wound healing. Biomacromolecules. 2011;12(8):2872-2880.

37. Miguel SP, Ribeiro MP, Brancal H, Coutinho P, Correia IJ. Thermoresponsive chitosan-agarose hydrogel for skin regeneration. Carbohydr Polym. 2014;111:366-373

38. Jahovic N, Güzel E, Arbak S, Yegen BC. The healing-promoting effect of saliva on skin burn is mediated by epidermal growth factor (EGF): role of the neutrophils. Burns. 2004;30(6):531-538.

39. Vaiana CA, Leonard MK, Drummy LF, et al. Epidermal growth factor: layered silicate nanocomposites for tissue regeneration. Biomacromolecules. 2011;12(9):3139-3146.

40. Pulat M, Kahraman AS, Tan N, Gümüşderelioğlu M. Sequential antibiotic and growth factor releasing chitosan-PAAm semi-IPN hydrogel as a novel wound dressing. J Biomater Sci Polym Ed. 2013;24(7): 807-819.

41. Hori K, Sotozono C, Hamuro J, et al. Controlled-release of epidermal growth factor from cationized gelatin hydrogel enhances corneal epithelial wound healing. J Control Release. 2007;118(2):169-176.

42. Chu Y, Yu D, Wang P, et al. Nanotechnology promotes the full-thickness diabetic wound healing effect of recombinant human epidermal growth factor in diabetic rats. Wound Repair Regen. 2010;18(5):499-505.

43. Phan TT, See P, Lee ST, Chan SY. Protective effects of curcumin against oxidative damage on skin cells in vitro: its implication for wound healing. J Trauma. 2001;51(5):927-931.

44. Kulac M, Aktas C, Tulubas F, et al. The effects of topical treatment with curcumin on burn wound healing in rats. J Mol Histol. 2013;44(1): 83-90.

45. Panchatcharam M, Miriyala S, Gayathri VS, Suguna L. Curcumin improves wound healing by modulating collagen and decreasing reactive oxygen species. Mol Cell Biochem. 2006;290(1-2):87-96.

46. Mani H, Sidhu GS, Kumari R, et al. Curcumin differentially regulates TGF- $\beta 1$, its receptors and nitric oxide synthase during impaired wound healing. Biofactors. 2002;16(1-2):29-43.

47. Mohanty C, Das M, Sahoo SK. Sustained wound healing activity of curcumin loaded oleic acid based polymeric bandage in a rat model. Mol Pharm. 2012;9(10):2801-2811.

48. Mitra T, Manna PJ, Raja ST, Gnanamani A, Kundu PP. Curcumin loaded nano graphene oxide reinforced fish scale collagen-a 3D scaffold biomaterial for wound healing applications. RSC Advances. 2015;5(119):98653-98665.

49. Gao X, Wang S, Wang BL, et al. Improving the anti-ovarian cancer activity of docetaxel with biodegradable self-assembly micelles through various evaluations. Biomaterials. 2015;53:646-658.

50. Wu Y, Chen L, Scott PG, Tredget EE. Mesenchymal stem cells enhance wound healing through differentiation and angiogenesis. Stem Cells. 2007;25(10):2648-2659. 


\section{Supplementary materials}

A<smiles>CC(O)COCCOC(C)CO</smiles>

b, c, d

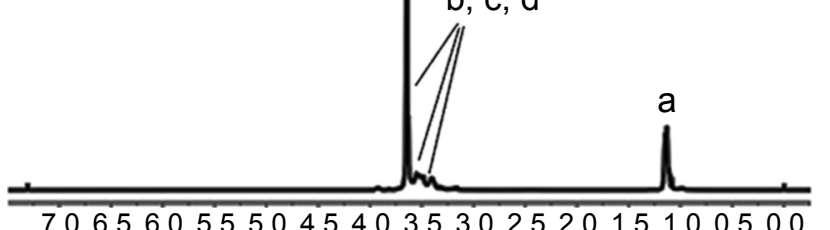

B<smiles>CC(COC(=O)C(C)O)OCCOCCO</smiles>

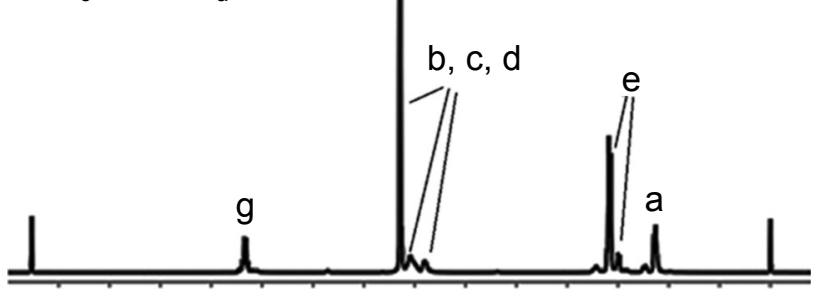

7.06 .56 .05 .55 .04 .54 .03 .53 .02 .52 .01 .51 .00 .50 .0

C

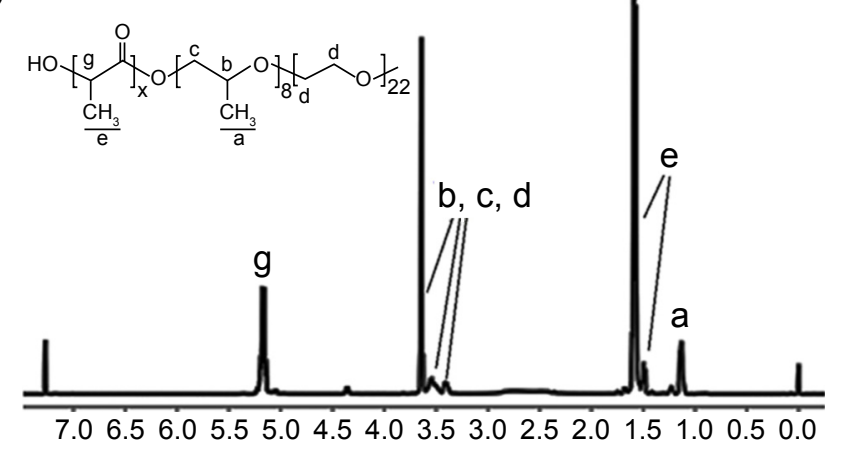

Figure SI 'H NMR test.

Notes: The 'H NMR spectrum of IOR5 (A); PLA-10R5-PLA, $M_{n}=4.6 \times 10^{3}$ (B); and PLA-10R5-PLA, $M_{n}=19.8 \times 10^{3}$ (C).

Abbreviations: 'H NMR, 'H nuclear magnetic resonance spectroscopy; PLA, polylactic acid; IOR5, reverse Pluronic ${ }^{\circledR}$ IOR5; $M_{n}$, number-average molecular weight.

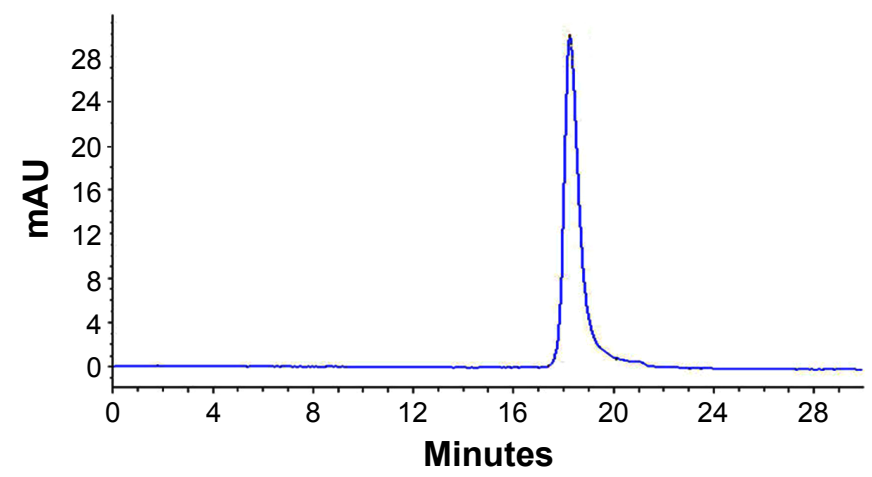

Figure $\mathbf{S 2}$ Analysis of EGF purity by HPLC.

Abbreviations: HPLC, high performance liquid chromatography; EGF, epidermal growth factor. 

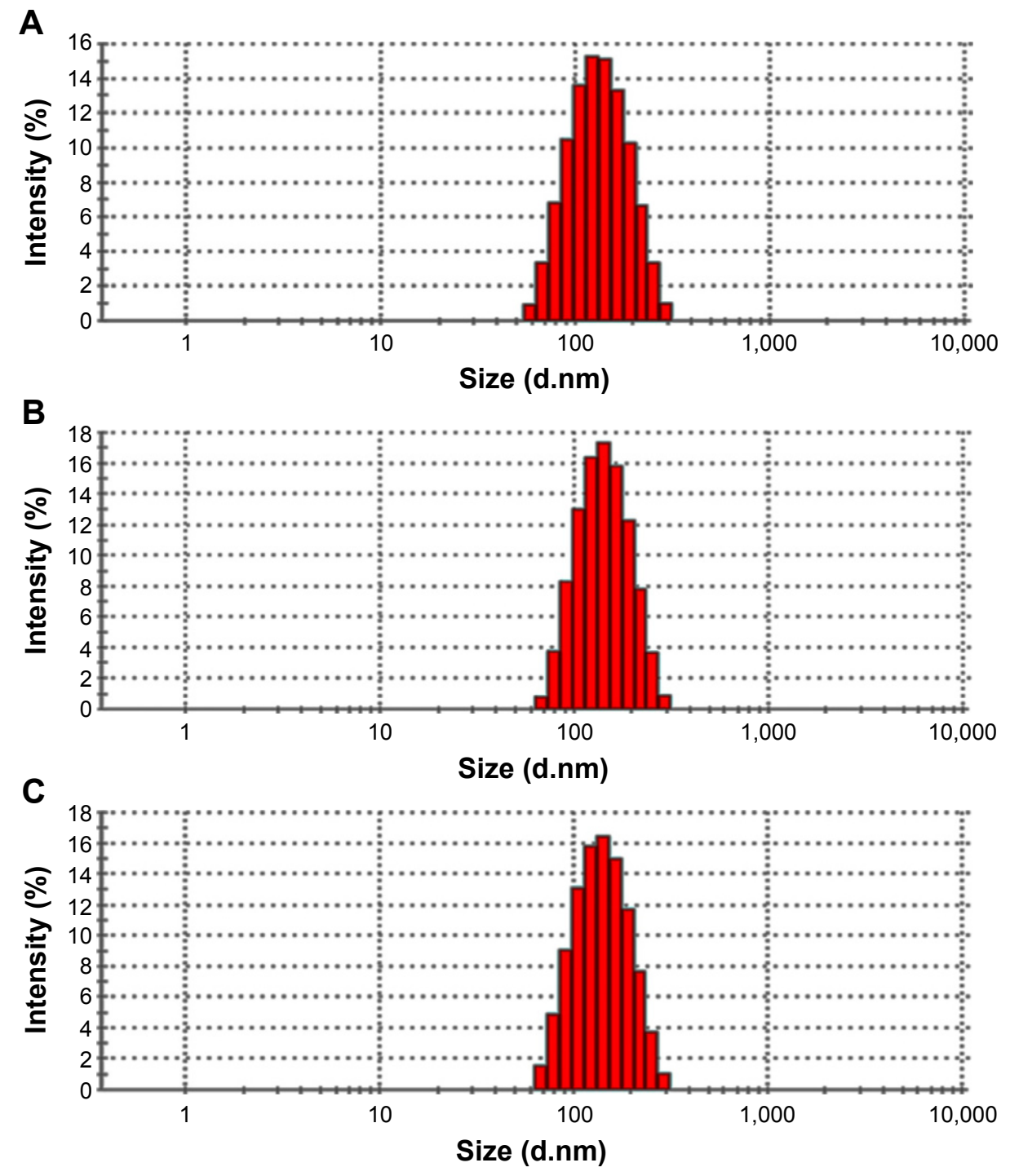

Figure S3 Particle size distribution of the nanoparticles.

Notes: Particle size distribution of the black NP (A); EGF-NP (B); and Cur-NP (C).

Abbreviations: NP, nanoparticle; Cur, curcumin; EGF, epidermal growth factor.

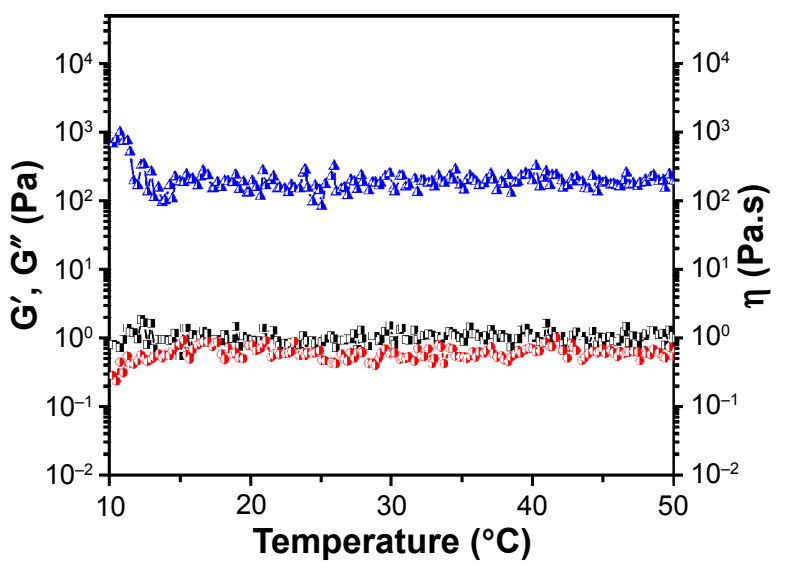

$$
\longrightarrow \mathrm{G}^{\prime} \longrightarrow \mathrm{G}^{\prime \prime} \longrightarrow \boldsymbol{\eta}
$$

Figure S4 Rheology analysis of reverse Pluronic ${ }^{\circledR}$ IOR5 aqueous solution (30 wt\%) as a function of temperature. Abbreviations: $G^{\prime}$, storage modulus; $G^{\prime \prime}$, loss modulus; $\eta$, viscosity. 
International Journal of Nanomedicine

Dovepress

\section{Publish your work in this journal}

The International Journal of Nanomedicine is an international, peerreviewed journal focusing on the application of nanotechnology in diagnostics, therapeutics, and drug delivery systems throughout the biomedical field. This journal is indexed on PubMed Central, MedLine, CAS, SciSearch ${ }^{\circledR}$, Current Contents ${ }^{\circledR} /$ Clinical Medicine,
Journal Citation Reports/Science Edition, EMBase, Scopus and the Elsevier Bibliographic databases. The manuscript management system is completely online and includes a very quick and fair peer-review system, which is all easy to use. Visit http://www.dovepress.com/ testimonials.php to read real quotes from published authors.

Submit your manuscript here: http://www.dovepress.com/international-journal-of-nanomedicine-journal 